\title{
Simultaneous Cyclic Scheduling and Control of a Multiproduct CSTR Reactor
}

\author{
Antonio Flores-Tlacuahuac* \\ Departamento de Ingeniería y Ciencias Químicas, Universidad Iberoamericana \\ Prolongación Paseo de la Reforma 880, México D.F., 01210, México \\ Ignacio E. Grossmann \\ Department of Chemical Engineering, Carnegie-Mellon University \\ 5000 Forbes Av., Pittsburgh 15213, PA
}

21st November 2005

*Author to whom correspondence should be addressed. E-mail: antonio.flores@uia.mx, phone/fax: +52(55)59504074, http://200.13.98.241/ antonio 


\begin{abstract}
In this work we propose a scheduling and control formulation for simultaneously addressing scheduling and control problems by explicitly incorporating process dynamics in the form of system constraints that ought to be met. The formulation takes into account the interactions between such problems and is able to cope with nonlinearities embedded into the processing system. The simultaneous scheduling and control problems is cast as a Mixed-Integer Dynamic Optimization (MIDO) problem where the simultaneous approach, based on orthogonal collocation on finite elements, is used to transform it into a Mixed-Integer Nonlinear Programming (MINLP) problem. The proposed simultaneous scheduling and control formulation is tested using three multiproduct continuous stirred tank reactors featuring hard nonlinearities.
\end{abstract}




\section{Introduction}

Traditionally, scheduling and control problems in chemical processes have been addressed in a separate way. From a scheduling point of view, the interest lies in determining optimal assignments to equipment production sequences, production times for each product, inventory levels that lead to maximum profit or minimum completion time [1]. Commonly, during this task, features related to the dynamic behavior of the underlying process are not taken into account. Similarly, when computing optimal transition trajectories (i.e. optimal values of the manipulated and controlled variables) between different set of products, one of the major objectives lies in determining the transition trajectory featuring minimum transition time [2]. When addressing optimal control problems, it is normally assumed that the production sequence is fixed [3]. Hence, normally scheduling features are neglected in optimal control formulations. In pure scheduling problems, normally the transition times between the different product combinations are fixed, and hence, the dynamic profile of the chosen manipulated and controlled variables is not taken into account in the optimization formulation.

However, it is recognized [4],[5],[6],[7] that scheduling and control problems are closely related problems and that, ideally, they should be addressed simultaneously rather than sequentially or, even worse, solved without taking account the other part (i.e. pure scheduling and control problems). Working along this line, the normal interactions between scheduling and control problems are taken into account, therefore leading to an improved objective function value and avoiding suboptimal solutions that occur when scheduling and control problems are addressed sequentially

Some early attempts to address the scheduling and control problem were made by Bathia and Biegler [3] who fixed the production sequence and used dynamic optimization for production planning optimization. Mahadevan et al. [4] analyzed grade transition scheduling problems from a robust closed-loop point of view. They did not address the problem as a MIDO problem, although it is recognized that optimal grade transition and scheduling problems should be approached along this line. They obtained grade 
schedulings by defining easy and hard to carry out transitions. Chatzidoukas et al. [8] proposed a MIDO formulation for analyzing polymer grade transition and optimal campaign scheduling. For solving the MIDO problem, they used the algorithm proposed by allgor [9]. Smania and Pinto [10] used steady-state models, and discrete time decisions, for optimizing production campaigns.

There have been more recents works addressing the scheduling and control problem. Mishra et al. [6] made a comparison between what they call the standard recipe approach (SRA) and the overall optimization approach (OOA) for solving SC problems. In the SRA approach process dynamics, trough the direct incorporation of a process mathematical model, is neglected and in its place a set of correlations, obtained from running local optimizations, are developed to capture time domain behavior. On the other hand, in the OOA approach, the process dynamic model is included into the formulation. The set of ordinary differential equations modeling the related process are discretized and transformed in to a set of algebraic equations. Hence, in the OOA method, the resulting SC problem is cast in terms of a MINLP problem. The authors claim that because in the OOA method the number of available freedom degrees is larger than in the SRA method, the optimal solution obtained by using the OOA method will be superior to the one obtained using the SRA method as their two cases of study show. Although the superiority of the OOA method for addressing SC problems is clear from their examples, they concluded that the use of the discretization approach to transform a MIDO problem into a MINLP problem is not feasible due to the large of number of constraints generated when discretizing the process dynamic model. Moreover, the authors solved the MINLP problems by a direct approach, meaning that they did not use any decomposition solution strategy aimed to reduce the computational complexity faced when solving MIDO problems. They concluded that additional work is needed to improve MIDO solution techniques.

In another recent work [7], scheduling and grade transition for polymerization systems has been addressed. The authors proposed a decomposition scheme specifically tailored for the resulting MIDO problem. Based on previous work about MIDO problem solution 
strategies [9] they proposed to solve the MIDO problem as sequence of primal and master problems. The primal problem contains the dynamic optimization part while the master problem deals with the scheduling part. The authors report good convergence properties when analyzing scheduling and grade transition for a polymerization plant, however no details of the specific polymerization system are offered. As the authors recognize, their MIDO solution strategy is highly application specific and therefore it would be difficult to apply even to other polymerization systems. Without enough details about the polymerization process dynamics and nonlinearities embedded, it turns out to be difficult to assess the robustness of the solution strategy.

In this work we propose a simultaneous approach to address scheduling and control problems for a continuous stirred tank reactor (CSTR) that produces multiple products. We take advantage of the rich knowledge of scheduling and optimal control formulations and we merge them so the the final result is a formulation able to solve simultaneous scheduling and control problems. We cast the problem as an optimization problem. In the proposed formulation, integer variables are used to determine the best production sequence and continuous variables take into account production times, cycle time and inventories. Because, dynamic profiles of both manipulated and controlled variables are also decision variables, the resulting problem is cast as a Mixed-Integer Dynamic Optimization (MIDO) problem. To solve the MIDO problem we use a recently proposed methodology [11] which consists in transforming the MIDO problem into a MINLP that can be solved using standard methods such as the Outer-Approximation method [12],[13]. Roughly speaking, the strategy for solving the MIDO problem consists in using the so-called Simultaneous Approach [2] for solving optimal control problems as the way to transform the set of ordinary differential equations modeling the dynamic system behavior into a set of algebraic equations. Because of the highly nonlinear behavior embedded in chemical process models, the resulting MIDO formulation will be a MINLP problem featuring hard nonlinearities such as multiple steady-states, parametric sensitivity, bifurcation and even chaotic dynamics. 


\section{Problem definition}

Given are a number of products that are to be manufactured in a single continuous multiproduct CSTR. Steady-state operating conditions for manufacturing each product are also specified, as well as the demand rate and price of each product and the inventory and raw materials costs. The problem to be tackled consists in the simultaneous determination of the best production wheel (i.e. cyclic time and the sequence in which the products will be manufactured) as well as the transition times, production rates, length of processing times, amounts manufactured of each product, such that the profit is maximized subject to a set of scheduling and dynamic state constraints.

\section{Scheduling and Control MIDO Formulation}

In the following simultaneous scheduling and control (SSC) formulation, we assume that all products are manufactured in a single CSTR and that the products follow a production wheel meaning that all the required products are manufactured, in an optimal sequence to be determined, and that the sequence is repeated cyclically (see Pinto and Grossmann [14] for the scheduling formulation). As shown in Figure 1(a) the cyclic time is divided into a series of slots. Within each slot two operations are carried out: (a) the production period during which a given product is manufactured around steady-state conditions and (b) the transition period during which dynamic transitions between two products take place. According to this description, Figure 1(b) depicts a typical dynamic operating response curve within each slot. At the beginning of each slot, material of a given product is manufactured until the demand imposed on such product is met, during this period both the system states $x$ and the manipulated variables $u$ remain constant. Afterwards, the CSTR process conditions are changed (by modifying the manipulated variables $u$ ) until new desired process operating conditions (as represented by the system states $x$ ), leading to the manufacture of a new product, are reached. In this work we assume that only one product can be produced in a slot and that each product is produced only once within 


\section{Transition period}

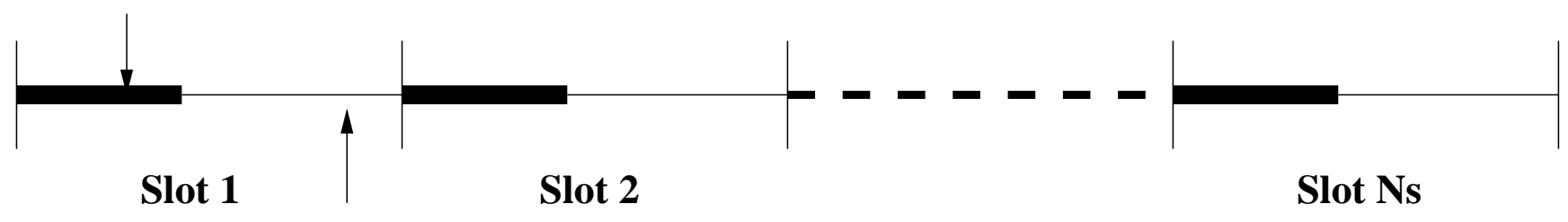

Production period

(a)

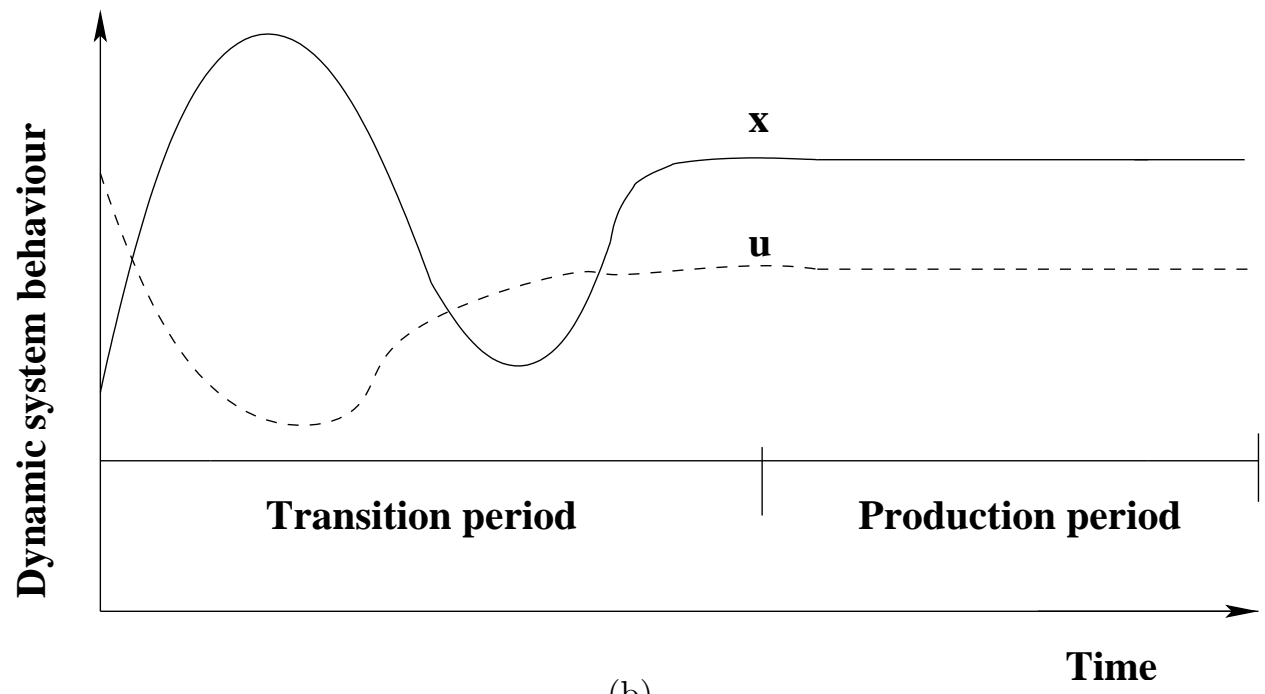

(b)

Figure 1: (a) The cyclic time is divided into slots and within each slot a steady-state production period is followed by a transition period. (b) Within each slot the system states $x$ and the manipulated variables $u$ remain constant. However during the transition period the manipulated variables change and so does the system states.

each production wheel. Also we assume that once a production wheel is completed, new identical cycles are executed indefinitely.

All the indices, decision variables and system parameters used in the SSC MIDO problem formulation are as follows:

1. Indices 
Products

Slots

Finite elements

Collocation points

System states

Manipulated variables $m=1, \ldots N_{u}$ $i, p=1, \ldots N_{p}$

$k=1, \ldots N_{s}$

$f=1, \ldots N_{f e}$

$c, l=1, \ldots N_{c p}$

$n=1, \ldots N_{x}$ 
2. Decision variables

$y_{i k} \quad$ Binary variable to denote if product $i$ is assigned to slot $k$

$y_{i k}^{\prime} \quad$ Binary auxiliary variable

$z_{i p k} \quad$ Binary variable to denote if product $i$ is followed by product $p$ in slot $k$

$p_{k} \quad$ Processing time at slot $k$

$t_{k}^{e} \quad$ Final time at slot $k$

$t_{k}^{s} \quad$ Start time at slot $k$

$G_{i} \quad$ Production rate

$T_{c} \quad$ Total production wheel time $[\mathrm{h}]$

$x_{f c k}^{n} \quad$ N-th system state in finite element $f$ and collocation point $c$ of slot $k$

$u_{f c k}^{m} \quad$ M-th manipulated variable in finite element $f$ and collocation point $c$ of slot $k$

$W_{i} \quad$ Amount produced of each product $[\mathrm{kg}]$

$\theta_{i k} \quad$ Processing time of product $i$ in slot $k$

$\theta_{k}^{t} \quad$ Transition time at slot $k$

$\Theta_{i} \quad$ Total processing time of product $i$

$x_{o, f k}^{n} \quad n$-th state value at the beginning of the finite element $f$ of slot $k$

$\bar{x}_{k}^{n} \quad$ Desired value of the $n$-th state at the end of slot $k$

$\bar{u}_{k}^{m} \quad$ Desired value of the $m$-th manipulated variable at the end of slot $k$

$x_{i n, k}^{n} \quad n$-th state value at the beginning of slot $k$

$u_{i n, k}^{n} \quad m$-th manipulated variable value at the beginning of slot $k$

$X_{i} \quad$ Conversion

3. Parameters

$N_{p} \quad$ Number of products

$N_{s} \quad$ Number of slots

$N_{f e} \quad$ Number of finite elements

$N_{c p} \quad$ Number of collocation points

$N_{x} \quad$ Number of system states

$N_{u} \quad$ Number of manipulated variables 


$\begin{array}{ll}D_{i} & \text { Demand rate }[\mathrm{kg} / \mathrm{h}] \\ C_{i}^{p} & \text { Price of products }[\$ / \mathrm{kg}] \\ C_{i}^{s} & \text { Cost of inventory } \\ C^{r} & \text { Cost of raw material } \\ h_{f k} & \text { Length of finite element } f \text { in slot } k \\ \Omega_{c c} & \text { Matrix of Radau quadrature weights } \\ \bar{x}_{k}^{n} & \text { Desired value of the } n \text {-th system state at slot } k \\ \bar{u}_{k}^{m} & \text { Desired value of the } m \text {-th manipulated variable at slot } k \\ \theta^{\text {max }} & \text { Upper bound on processing time } \\ t_{i p}^{t} & \text { Estimated value of the transition time between product } i \text { and } p \\ x_{s s, i}^{n} & n \text {-th state steady value of product } i \\ u_{s s, i}^{m} & m \text {-th manipulated variable value of product } i \\ F^{o} & \text { Feed stream volumetric flow rate } \\ X_{i} & \text { Conversion degree } \\ x_{\text {min }}^{n}, x_{\max }^{n} & \text { Minimum and maximum value of the state } x^{n} \\ u_{\min }^{m}, u_{\max }^{m} & \text { Minimum and maximum value of the manipulated variable } u^{m} \\ \gamma_{c} & \text { Roots of the Lagrange orthogonal polynomial } \\ \end{array}$

In order to clarify the SSC MIDO problem formulation, it has been divided into two parts. The first one deals with the scheduling part and the second one with the dynamic optimization part.

\section{- Objective function.}

$$
\begin{aligned}
\max & \left\{\sum_{i=1}^{N_{p}} \frac{C_{i}^{p} W_{i}}{T_{c}}-\sum_{i=1}^{N_{p}} \frac{C_{i}^{s}\left(G_{i}-W_{i}\right)}{2 \Theta_{i} T_{c}}-\sum_{k=1}^{N_{s}} \sum_{f=1}^{N_{f e}} h_{f k} \sum_{c=1}^{N_{c p}} \frac{C^{r} t_{f c k} \Omega_{c, N_{c p}}}{T_{c}}\left(\left(x_{f c k}^{1}-\bar{x}_{k}^{1}\right)^{2}\right.\right. \\
& \left.\left.+\ldots+\left(x_{f c k}^{n}-\bar{x}_{k}^{n}\right)^{2}+\left(u_{f c k}^{1}-\bar{u}_{k}^{1}\right)^{2}+\ldots+\left(u_{f c k}^{m}-\bar{u}_{k}^{m}\right)^{2}\right)\right\}
\end{aligned}
$$

The total process profit is given by the amount and cost of the manufactured products minus the sum of the inventory costs and the product transition costs. As a measure of 
the transition costs, we use a term that takes into account the amount of off-specification material produced during product transition. At each slot, such term would have the following form:

$$
\frac{1}{T_{c}} \int_{0}^{t_{f}}\left[\sum_{n}\left(x^{n}-\bar{x}^{n}\right)^{2}+\sum_{m}\left(u^{m}-\bar{u}^{m}\right)^{2}\right] C^{r} d t
$$

where $t_{f}$ is the transition time in slot $k, C^{r}$ is the cost of the raw material, $T_{c}$ is duration of the production wheel cycle, $x^{n}$ is the $n$-th system state and $\bar{x}^{n}$ its desired value. Similarly, $u^{m}$ is the $m$-th manipulated variable and $\bar{u}^{m}$ its desired value. The above integral can be approximated by Radau quadrature as follows:

$\sum_{k=1}^{N_{s}} \sum_{f=1}^{N_{f e}} h_{f k} \sum_{c=1}^{N_{p c}} \frac{C^{r} t_{f c k} \Omega_{c, N_{c p}}}{T_{c}}\left(\left(x_{f c k}^{1}-\bar{x}_{k}^{1}\right)^{2}+\ldots+\left(x_{f c k}^{n}-\bar{x}_{k}^{n}\right)^{2}+\left(u_{f c k}^{1}-\bar{u}_{k}^{1}\right)^{2}+\ldots+\left(u_{f c k}^{m}-\bar{u}_{k}^{m}\right)^{2}\right)$

It should be noted that this form of the transition costs will force the system to carry out product transitions as soon as possible, while at the end of a product transition the states will take the steady-state values for manufacturing a new product.

1. Scheduling part.

a) Product assignment

$$
\begin{aligned}
\sum_{k=1}^{N_{s}} y_{i k} & =1, \forall i \\
\sum_{i=1}^{N_{p}} y_{i k} & =1, \forall k \\
y_{i k}^{\prime} & =y_{i, k-1}, \forall i, k \neq 1 \\
y_{i, 1}^{\prime} & =y_{i, N_{s}}, \forall i
\end{aligned}
$$

Equation 3a states that, within each production wheel, any product can only be manufactured once, while constraint $3 \mathrm{~b}$ implies that only one product is 
manufactured at each slot. Due to this constraint, the number of products and slots turns out to be the same. Equation 3c defines backward binary variable $\left(y_{i k}^{\prime}\right)$ meaning that such variable for product $i$ in slot $k$ takes the value assigned to the same binary variable but one slot backwards $k-1$. At the first slot, Equation 3d defines the backward binary variable as the value of the same variable at the last slot. This type of assignment reflects our assumption of cyclic production wheel. The variable $y_{i k}^{\prime}$ will be used later to determine the sequence of product transitions.

b) Amounts manufactured

$$
\begin{aligned}
& W_{i} \geqslant D_{i} T_{c}, \forall i \\
& W_{i}=G_{i} \Theta_{i}, \forall i \\
& G_{i}=F^{o}\left(1-X_{i}\right), \forall i
\end{aligned}
$$

Equation 4a states that the total amount manufactured of each product $i$ must be equal or greater than the desired demand rate times the duration of the production wheel, while Equation $4 \mathrm{~b}$ indicates that the amount manufactured of product $i$ is computed as the product of the production rate $\left(G_{i}\right)$ times the time used $\left(\Theta_{i}\right)$ for manufacturing such product. The production rate is computed from Equation $4 \mathrm{c}$ as a simple relationship between the feed stream flowrate $\left(F^{o}\right)$ and the conversion $\left(X_{i}\right)$.

c) Processing times

$$
\begin{aligned}
\theta_{i k} & \leqslant \theta^{\text {max }} y_{i k}, \forall i, k \\
\Theta_{i} & =\sum_{k=1}^{N_{s}} \theta_{i k}, \forall i \\
p_{k} & =\sum_{i=1}^{N_{p}} \theta_{i k}, \forall k
\end{aligned}
$$

The constraint given by Equation 5a sets an upper bound on the time used for 
manufacturing product $i$ at slot $k$. Equation $5 \mathrm{~b}$ is the time used for manufacturing product $i$, while Equation 5 c defines the duration time at slot $k$.

d) Transitions between products

$$
z_{i p k} \geqslant y_{p k}^{\prime}+y_{i k}-1, \forall i, p, k
$$

The constraint given in Equation 6 is used for defining the binary production transition variable $z_{i p k}$. If such variable is equal to 1 then a dynamic transition will occur from product $i$ to product $p$ within slot $k, z_{i p k}$ will be zero otherwise.

e) Timing relations

$$
\begin{aligned}
\theta_{k}^{t} & =\sum_{i=1}^{N_{p}} \sum_{p=1}^{N_{p}} t_{p i}^{t} z_{i p k}, \forall k \\
t_{1}^{s} & =0 \\
t_{k}^{e} & =t_{k}^{s}+p_{k}+\sum_{i=1}^{N_{p}} \sum_{p=1}^{N_{p}} t_{p i}^{t} z_{i p k}, \forall k \\
t_{k}^{s} & =t_{k-1}^{e}, \forall k \neq 1 \\
t_{k}^{e} & \leqslant T_{c}, \forall k \\
t_{f c k} & =(f-1) \frac{\theta_{k}^{t}}{N_{f e}}+\frac{\theta_{k}^{t}}{N_{f e}} \gamma_{c}, \forall f, c, k
\end{aligned}
$$

Equation $7 \mathrm{a}$ defines the transition time from product $i$ to product $p$ at slot $k$. It should be remarked that the term $t_{p i}^{t}$ stands only for an estimate of the expected transition times. Because such transition times depend upon process dynamic behavior, they will be computed as part of the scheduling and control formulation. Good estimates of the transition times can be obtained from openloop dynamic optimization runs between all pairs of products. If this terms happens to be difficult to evaluate, or the number of combinations of product schedules turns out to be large, $t_{p i}^{t}$ values could be set as 4 times the reactor open-loop residence time. In any case, transition times are obtained iteratively. First, one needs to guess transition time values $\left(t_{p i}^{t}\right)$, solve the MIDO problem 
and check if the computed transition time values $\left(\theta_{k}^{t}\right)$ are long enough to allow safe and smooth grade transition dynamic behavior. Frequently, large dynamic variations in the states and manipulated variables behavior are an indication that by increasing the guessed $t_{p i}^{t}$ values, better and smoother dynamic grade transition behavior could be obtained. Normally, in a few number of iterations, one can easily obtain acceptable grade transition dynamic behavior. Equation $7 \mathrm{~b}$ sets to zero the time at the beginning of the production wheel cycle corresponding to the first slot. Equation $7 \mathrm{c}$ is used for computing the time at the end of each slot as the sum of the slot start time plus the processing time and the transition time. Equation $7 d$ states that the start time at all the slots, different than the first one, is just the end time of the previous slot. Equation 7e is used to force that the end time at each slot be less than the production wheel cyclic time. Finally, Equation $7 \mathrm{f}$ is used to obtain the time value inside each finite element and for each internal collocation point.

\section{Dynamic Optimization part.}

To address the optimal control part, the so-called simultaneous approach [2] for solving dynamic optimization problems was used. In this approach the dynamic mathematical model representing system behavior is discretized using the method of orthogonal collocation on finite elements [15],[16]. According to this procedure, a given slot $k$ is divided into a number of finite elements. Within each finite element an adequate number of internal collocation points is selected as depicted in Figure 2. Using several finite elements is useful to represent dynamic profiles with nonsmooth variations. Thereby, the set of ordinary differential equations comprising the system model, is approximated at each collocation point leading to a set of nonlinear equations that must be satisfied. 


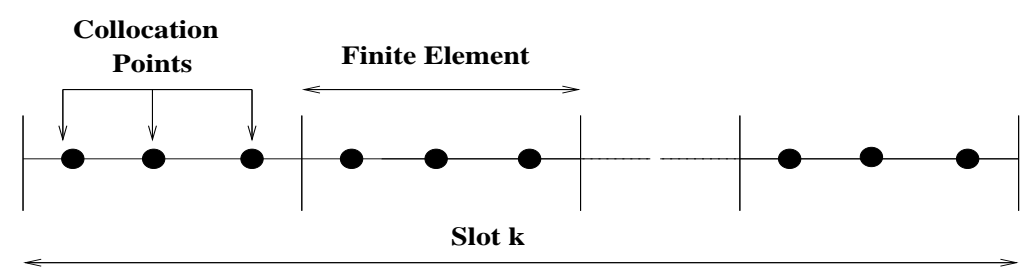

Figure 2: Simultaneous discretization approach for dealing with dynamic optimization problems. Each slot $k$ is divided into $N_{f e}$ finite elements. Within each finite element $f$ a set of $N_{c p}$ collocation points $c$ is selected.

a) Dynamic mathematical model discretization

$$
x_{f c k}^{n}=x_{o, f k}^{n}+\theta_{k}^{t} h_{f k} \sum_{l=1}^{N_{c p}} \Omega_{l c} \dot{x}_{f l k}^{n}, \forall n, f, c, k
$$

The constraints given by Equations 8 are used to compute the value of the system states at each one of the discretized points $\left(x_{f c k}^{n}\right)$ by using the monomial basis representation. $x_{o, f k}^{n}$ is the $n$-th system state at the beginning of each element, $\Omega_{l c}$ is the collocation matrix and $\dot{x}_{f c k}^{n}$ is the first order derivative of the $n$-th state. Notice that when working with the first element $x_{o, 1 k}^{n}$ represents the specified initial value of the $n$-th state. Also notice that in the present formulation the length of all finite elements is the same and computed as

$$
h_{f k}=\frac{1}{N_{f e}}
$$

b) Continuity constraint between finite elements

$$
x_{o, f k}^{n}=x_{o, f-1, k}^{n}+\theta_{k}^{t} h_{f-1, k} \sum_{l=1}^{N_{c p}} \Omega_{l, N_{c p}} \dot{x}_{f-1, l, k}^{n}, \forall n, f \geqslant 2, k
$$

In the simultaneous approach for dynamic optimization problems, only the states must be continuous when crossing from one given finite element to the next one; algebraic and manipulated variables are allowed to exhibit discontinuity behavior between adjacent finite elements. That is the reason why continuity constraints are not formulated for algebraic and manipulated vari- 
ables. We use Equations 10 to force continuous state profiles on all the elements at the beginning of each element $\left(x_{o, f k}^{n}\right)$ and they are computed in terms of the same monomial basis used before for defining the value of the system states.

c) Model behavior at each collocation point

$$
\dot{x}_{f c k}^{n}=f^{n}\left(x_{f c k}^{1}, \ldots, x_{f c k}^{n}, u_{f c k}^{1}, \ldots u_{f c k}^{m}\right), \forall n, f, c, k
$$

Equations 11 are used for computing the value of the first order derivatives of the systems at finite element $f$ of collocation point $c$ in slot $k$. Those equations simply represent the right hand sides of the dynamic model. Because our scheduling and control formulation is system independent, we have used the notation $f^{n}$ to represent the right hand side of the $n$-th ordinary differential equation describing any desired dynamic system. 
d) Initial and final controlled and manipulated variable values at each slot

$$
\begin{aligned}
x_{i n, k}^{n} & =\sum_{i=1}^{N_{p}} x_{s s, i}^{n} y_{i, k}, \forall n, k \\
\bar{x}_{k}^{n} & =\sum_{i=1}^{N_{p}} x_{s s, i}^{n} y_{i, k+1}, \forall n, k \neq N_{s} \\
\bar{x}_{k}^{n} & =\sum_{i=1}^{N_{p}} x_{s s, i}^{n} y_{i, 1}, \forall n, k=N_{s} \\
u_{i n, k}^{m} & =\sum_{i=1}^{N_{p}} u_{s s, i}^{m} y_{i, k}, \forall m, k \\
\bar{u}_{k}^{m} & =\sum_{i=1}^{N_{p}} u_{s s, i}^{m} y_{i, k+1}, \forall m, k \neq N_{s}-1 \\
\bar{u}_{k}^{m} & =\sum_{i=1}^{N_{p}} u_{s s, i}^{m} y_{i, 1}, \forall m, k=N_{s} \\
x_{N_{f e}, N_{c p}, k}^{n} & =\bar{x}_{k}^{n}, \forall n, k \\
u_{1,1, k}^{m} & =u_{i n, k}^{m}, \forall m, k \\
u_{N_{f e}, N_{c p}, k}^{m} & =\bar{u}_{i n, k}^{m}, \forall m, k \\
x_{o, 1, k}^{n} & =x_{i n, k}^{n}, \forall n, k
\end{aligned}
$$

Equations 12 define the values of the state variables at the beginning of each slot $k\left(x_{i n, k}^{n}\right)$. The desired value of each state at the end of the same slot $k\left(\bar{x}_{k}^{n}\right)$ is computed in Equations 13-14. It should be stressed that the state values at the beginning and end of each slot $k$ are given by the corresponding steadystate values $\left(x_{s s, i}^{n}\right)$ calculated a priori. $x_{s s, i}^{n}$ simply stands for the steady-state value of the manufacturing product $i$. They can be easily obtained from openloop steady-state simulation of the processing system. Similarly, Equations 15-17 define the values of the manipulated variables at the beginning of each slot $k\left(u_{i n, k}^{m}\right)$ and at the end of the slot $k\left(\bar{u}_{k}^{m}\right)$. Equations 18 enforce the system states to take the desired state values at each slot $k$. A similar situation occurs with the values of the manipulated variables. Equations 19 fix the values at 
the first finite element and first collocation point of each slot $k\left(u_{1,1, k}^{m}\right)$ as the value that such variable takes at the beginning of the same slot $k$. Equations 20 determine the values of the manipulated variables at the last finite element and last collocation point of slot $k\left(u_{N_{f e}, N_{c p}, k}^{m}\right)$ as the desired steady-state value of the same variable at slot $k\left(\bar{u}_{k}^{m}\right)$. Finally, Equations 21 determine the values of the system states at the beginning of each slot $\left(x_{o, 1, k}^{n}\right)$.

e) Lower and upper bounds on the decision variables

$$
\begin{aligned}
& x_{\text {min }}^{n} \leqslant x_{f c k}^{n} \leqslant x_{\text {max }}^{n}, \forall n, f, c, k \\
& u_{\text {min }}^{m} \leqslant u_{f c k}^{m} \leqslant u_{\text {max }}^{m}, \forall m, f, c, k
\end{aligned}
$$

Equations 22a-22b simply constrain the values of both the system states and manipulated variables to lie within acceptable lower and upper bounds.

\section{Case Studies}

In order to test the proposed simultaneous scheduling and control formulation three case studies, with different number of products and different degrees of nonlinear behavior embedded in the model, were addressed. In all the cases, CSTRs were used to manufacture the desired products. The case studies range from CSTRs featuring quasi-linear behavior to CSTRs with input multiplicities (second case study) and output multiplicities (third case study). In all the cases with nonlinear behavior the operating conditions were chosen around nonlinear behavior regions. We did so because most chemical processes featuring optimality conditions tend to exhibit regions of highly nonlinear behavior [17] and to have an exact idea about the complexities of solving MIDO problems with embedded nonlinearities. Hopefully, this will allow us to identify research areas where MIDO formulations/algorithms require improvements. 


\begin{tabular}{|cccccc|}
\hline Product & $\begin{array}{c}Q \\
{[\mathrm{lt} / \mathrm{hr}]}\end{array}$ & $\begin{array}{c}C_{R} \\
{[\mathrm{~mol} / \mathrm{lt}]}\end{array}$ & $\begin{array}{c}\text { Demand } \\
\text { rate }[\mathrm{Kg} / \mathrm{h}]\end{array}$ & $\begin{array}{c}\text { Product } \\
\text { cost }[\$ / \mathrm{kg}]\end{array}$ & $\begin{array}{c}\text { Inventory } \\
\text { cost }[\$]\end{array}$ \\
\hline$A$ & 10 & 0.0967 & 3 & 200 & 1 \\
$B$ & 100 & 0.2 & 8 & 150 & 1.5 \\
$C$ & 400 & 0.3032 & 10 & 130 & 1.8 \\
$D$ & 1000 & 0.393 & 10 & 125 & 2 \\
$E$ & 2500 & 0.5 & 10 & 120 & 1.7 \\
\hline
\end{tabular}

Table 1: Process data for the first case study. $A, B, C, D$ and $E$ stand for the five products to be manufactured.

\subsection{CSTR with a simple irreversible reaction}

The following reaction,

$$
R \stackrel{k}{\rightarrow} P,-\mathcal{R}_{R}=k C_{R}^{3}
$$

takes place in an isothermal CSTR for manufacturing 5 products $A, B, C, D, E$. The dynamic composition model is given by,

$$
\frac{d C_{R}}{d t}=\frac{Q}{V}\left(C_{o}-C_{R}\right)+\mathcal{R}_{R}
$$

where $\mathrm{Q}$ is the control variable for the dynamic transition in the production of one product to another. Using the following values of the design and kinetic parameters: $C_{o}=1 \mathrm{~mol} / \mathrm{lt}$, $V=5000 \mathrm{lt}, k=2 \mathrm{lt}^{2} /\left(\mathrm{mol}^{2}-\mathrm{h}\right)$, and the five values of the volumetric flowrate $Q$ shown in Table 1, the concentration five steady-states $C_{R}$, shown in the same table, are obtained. This table also contains information regarding the demand rate, products and inventory costs that are involved.

Solving the MIDO scheduling and control problem using GAMS/DICOPT, the optimizer selects the cyclic $A \rightarrow E \rightarrow D \rightarrow C \rightarrow B$ production wheel as the one which maximizes the profit. The objective function value turned out to be $\$ 7889$, while the total cycle time with the the sequence $A \rightarrow E \rightarrow D \rightarrow C \rightarrow B$ was 124.8 h. Additional information concerning processing times at each slot, production rates, total amounts of each product, transition times and initial and ending times at each slot are shown in Table 2. Regarding the dynamic behavior of the reactor during product transitions, Figure 3 


\begin{tabular}{|cccccccc|}
\hline Slot & Product & $\begin{array}{c}\text { Process time } \\
{[\mathrm{h}]}\end{array}$ & $\begin{array}{c}\text { Production rate } \\
{[\mathrm{Kg} / \mathrm{h}]}\end{array}$ & $\begin{array}{c}w \\
{[\mathrm{Kg}]}\end{array}$ & $\begin{array}{c}\text { Transition Time } \\
{[\mathrm{h}]}\end{array}$ & $\begin{array}{c}\text { T start } \\
{[\mathrm{h}]}\end{array}$ & $\begin{array}{c}\text { T end } \\
{[\mathrm{h}]}\end{array}$ \\
\hline 1 & $A$ & 41.5 & 9.033 & 374.31 & 5 & 0 & 46.4 \\
2 & $E$ & 23.3 & 1250 & 29162.3 & 5 & 46.4 & 74.7 \\
3 & $D$ & 2.06 & 607 & 1247.7 & 5 & 74.7 & 81.8 \\
4 & $C$ & 4.48 & 278.72 & 1247.7 & 5 & 81.8 & 91.2 \\
5 & $B$ & 12.48 & 80 & 998.2 & 21 & 91.2 & 124.7 \\
\hline
\end{tabular}

Table 2: Simultaneous scheduling and control results for the first case study. The objective function value is $\$ 7889$ and $124.8 \mathrm{~h}$ of total cycle time.

displays the dynamic profiles of both the manipulated variable $(Q)$ and the controlled variable $\left(C_{R}\right)$.

Looking at the results shown in Table 3, it turns out that the SSC formulation decided first to manufacture product $A$ because it is the most valuable product. In fact, the process time allocated for this product is also the larger one. However, the total amount manufactured of product $A(w)$ is not the larger one because the feed stream volumetric flow rate is the lowest one. We should remember that this is so because product $A$ has the higher conversion degree but it requires longer residence times to produce high conversion products. Because we assume constant reactor volume, the only way to get high residence times is allowing $Q$ to be small giving rise to low production levels. After completing the manufacture of product $A$, the optimizer decided to carry out a transition to product $E$, the less valuable product (later we will discuss that process dynamics, instead of process economics, dictates this transition). However, because for manufacturing product $E$ a small residence time is required, we can use larger feed stream volumetric flow rates getting higher production rates but, of course, of a product stream which still contains a significant portion of reactant. In fact, product $E$ is manufactured in the largest amount using the second largest process time. Starting from product $E$ the optimizer moves always towards more valuable products $(D, C, B)$ of increasing reactant conversion. However, because residence times tend to increase along the production sequence, the feed stream volumetric flow rate decreases and so does the total amount manufactured of each one of these products and the production rate. 

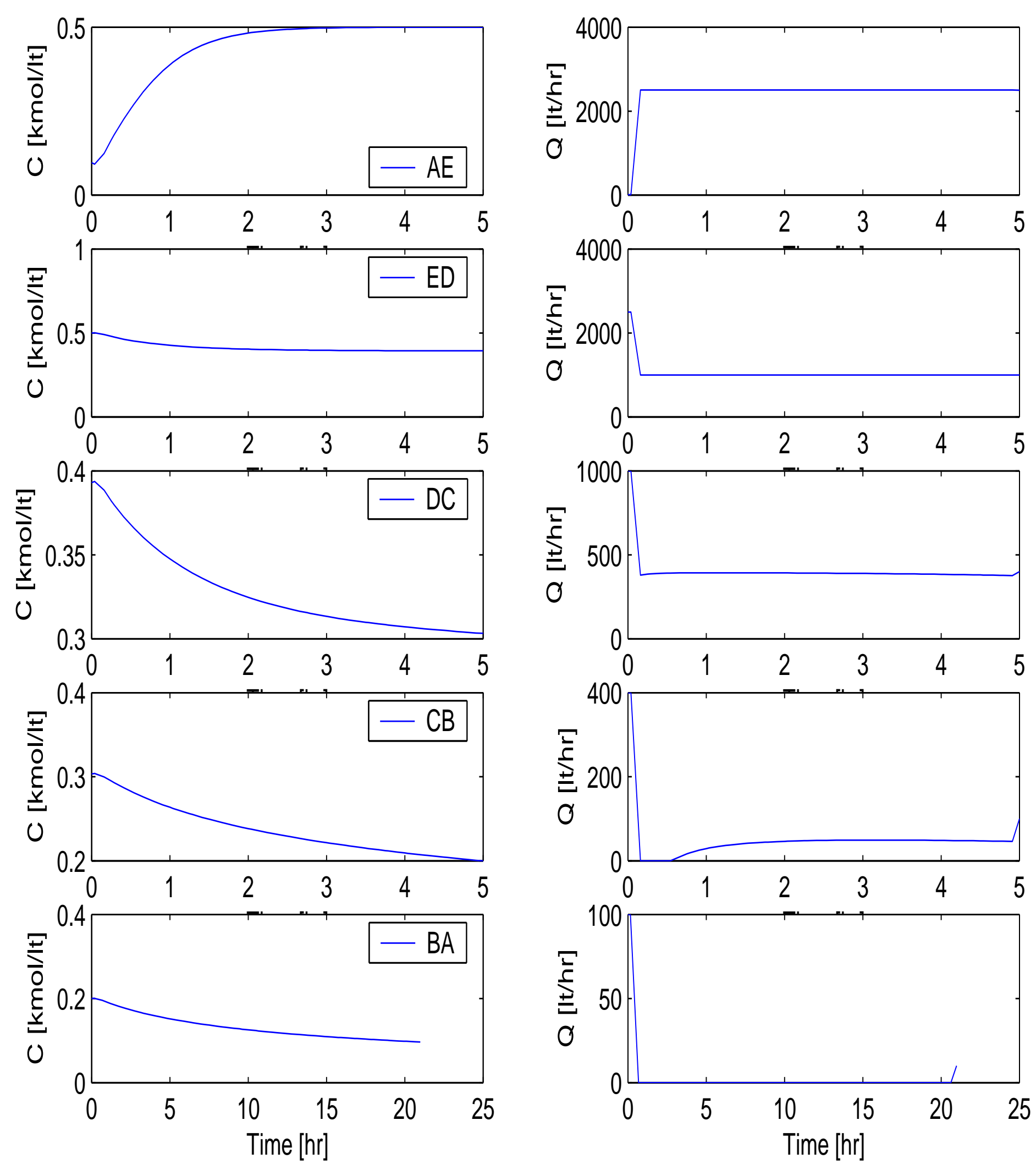

Figure 3: Optimal dynamic profiles for the volumetric flow rate and reactor concentration during product transition for the first case study. 
To understand why the optimizer selected the cyclic $A \rightarrow E \rightarrow D \rightarrow C \rightarrow B$ production sequence as the optimal one, we analyze the process dynamic behavior. If we obtain the transfer function, around each one of the process steady-states shown in Table 2, using $C_{R}$ and $Q$ as the controlled and manipulated variables, respectively

$$
G(s)=\frac{b}{s+a}
$$

where

$$
\begin{aligned}
b & =\frac{1}{V}\left(C_{o}-C_{R}\right) \\
a & =3 k C_{R}^{2}
\end{aligned}
$$

hence, from a process dynamics point of view, the process looks well behaved since it will be always stable, minimum-phase and, because it is a first order system, it will never display oscillatory behavior. For products $(A, B, C, D, E)$ the open loop poles and time constants are $(0.0561,0.24,0.5116,0.9267,1.5)$ and $(111.98,26.18,11.4,6.7,4.2)$, respectively. It is a well known fact that state transitions from small to large poles are faster than in the opposite direction because as the poles are located away from the imaginary axis, the system response speeds up. For open-loop system a state transition from a small to a large pole will take a longer time because the system dynamics turns out to be sluggish as the system poles are located closer to the imaginary axis. Using these ideas it now becomes clear why the optimizer picked up the cyclic $A \rightarrow E \rightarrow D \rightarrow C \rightarrow B$ production sequence. At the beginning the optimizer selected product $A$ simply because it is the most valuable one. Then, it decided to switch to product $E$ because the transition time from product $A$ to prodict $E$ is relatively small. From here, the quickest transitions are to product $D$, then to product $C$ and finally to product $B$. In table 2 we see that for all those product transitions, the transitions times are always $5 \mathrm{~h}$. At the end of the production wheel the system must start again a new production wheel which implies a transition $B \rightarrow A$ between the first and second production wheels. In terms of process 
dynamics, the transition times between two given open-loop stable products $P_{1}$ and $P_{2}$, with $\left|\lambda\left(P_{2}\right)\right|>\left|\lambda\left(P_{1}\right)\right|$ (where $\lambda$ stands for the eigenvalue of a given product), will tend to decrease as $\left|\lambda\left(P_{2}\right)\right| \rightarrow\left|\lambda\left(P_{1}\right)\right|$. Therefore, if we are interested in minimizing the transition time of the production sequence, then we should select products featuring open-loop poles as close as possible. This explains why, starting from product $E$, the optimizer decides to pick product $D$ instead of product $B$.

It is interesting to compare the optimal MIDO solution against the second and third best cyclic solutions. The second best solution is in fact a slight variation of the previous one. In this case the optimizer selects the cyclic $A \rightarrow D \rightarrow E \rightarrow C \rightarrow B$ processing sequence. To learn the reasons why the first production sequence turns out to be better than the second one, we have partitioned the objective function in three terms: $\phi_{1}$ dealing with the product profits, $\phi_{2}$ which deals with the inventory costs and $\phi_{3}$ dealing with the transition costs and defined as follows,

$$
\begin{aligned}
\phi_{1}= & \sum_{i=1}^{N_{p}} \frac{C_{i}^{p} W_{i}}{T_{c}} \\
\phi_{2}= & \sum_{i=1}^{N_{p}} \frac{C_{i}^{s}\left(G_{i}-W_{i}\right)}{2 \Theta_{i} T_{c}} \\
\phi_{3}= & \sum_{k=1}^{N_{s}} \sum_{f=1}^{N_{f e}} h_{f k} \sum_{c=1}^{N_{c p}} \frac{C^{r} t_{f c k} \Omega_{c, N_{c p}}}{T_{c}}\left(\left(x_{f c k}^{1}-\bar{x}_{k}^{1}\right)^{2}+\ldots+\right. \\
& \left.\left(x_{f c k}^{n}-\bar{x}_{k}^{n}\right)^{2}+\left(u_{f c k}^{1}-\bar{u}_{k}^{1}\right)^{2}+\ldots+\left(u_{f c k}^{m}-\bar{u}_{k}^{m}\right)^{2}\right)
\end{aligned}
$$

hence, the $\left[\phi_{1}, \phi_{s}, \phi_{3}\right]$ values are $[32397,23262,1247]$ and $[32463,23330,1234]$ for the first and second solutions, respectively (see Table 3 for information regarding optimal values of the additional decision variables). From this information, we see that both solutions have similar $\phi_{1}$ and $\phi_{2}$ values. However, the difference between those solutions is the transition cost: the second solution features a larger transition costs and this makes it suboptimal compared to the first one. Dynamic grade transitions for this production sequence are depicted in Figure 4. As can be seen, the dynamic grade transitions feature a shape that resembles the results of the best MIDO solution. 


\begin{tabular}{|cccccccc|}
\hline Slot & Product & $\begin{array}{c}\text { Process time } \\
{[\mathrm{h}]}\end{array}$ & $\begin{array}{c}\text { Production rate } \\
{[\mathrm{Kg} / \mathrm{h}]}\end{array}$ & $\begin{array}{c}w \\
{[\mathrm{Kg}]}\end{array}$ & $\begin{array}{c}\text { Transition Time } \\
{[\mathrm{h}]}\end{array}$ & $\begin{array}{c}\text { T start } \\
{[\mathrm{h}]}\end{array}$ & $\begin{array}{c}\text { T end } \\
{[\mathrm{h}]}\end{array}$ \\
\hline 1 & $A$ & 41.5 & 9.033 & 374.31 & 5 & 0 & 46.4 \\
2 & $D$ & 2.06 & 607 & 1249.4 & 5 & 46.4 & 53.6 \\
3 & $E$ & 23.4 & 1250 & 29270.4 & 5 & 53.6 & 82 \\
4 & $C$ & 4.48 & 278.72 & 1249.4 & 5 & 82 & 91.5 \\
5 & $B$ & 12.48 & 80 & 999.5 & 21 & 91.5 & 125 \\
\hline
\end{tabular}

Table 3: Simultaneous scheduling and control results for the first case study, second best solution. The objective function value is $\$ 7791$ and $125 \mathrm{~h}$ of total cycle time.

\begin{tabular}{|cccccccc|}
\hline Slot & Product & $\begin{array}{c}\text { Process time } \\
{[\mathrm{h}]}\end{array}$ & $\begin{array}{c}\text { Production rate } \\
{[\mathrm{Kg} / \mathrm{h}]}\end{array}$ & $\begin{array}{c}w \\
{[\mathrm{Kg}]}\end{array}$ & $\begin{array}{c}\text { Transition Time } \\
{[\mathrm{h}]}\end{array}$ & $\begin{array}{c}\text { T start } \\
{[\mathrm{h}]}\end{array}$ & $\begin{array}{c}\text { T end } \\
{[\mathrm{h}]}\end{array}$ \\
\hline 1 & $B$ & 12.7 & 80 & 1012.5 & 21 & 0 & 33.7 \\
2 & $A$ & 42.04 & 9.033 & 379.7 & 5 & 33.7 & 80.7 \\
3 & $E$ & 23.3 & 1250 & 29125.4 & 5 & 80.7 & 109 \\
4 & $C$ & 4.6 & 278.72 & 1265.6 & 5 & 109 & 118.6 \\
5 & $D$ & 2.09 & 607 & 1265.6 & 6 & 118.6 & 127 \\
\hline
\end{tabular}

Table 4: Simultaneous scheduling and control results for the first case study, third best solution. The objective function value is $\$ 6821.6$ and $127 \mathrm{~h}$ of total cycle time.

Regarding the third best MIDO optimal solution, the optimizer selected the cyclic $B \rightarrow A \rightarrow E \rightarrow C \rightarrow D$ production sequence. Information about the decision variables of this solution can be found in Table 4. As we can see, the third optimal solution has a larger objective function value decrease compared to the second one. Analyzing the $\left[\phi_{1}, \phi_{2}, \phi_{3}\right]=[31967,23352,1794]$ values we notice that the third solution features a decrease in $\phi_{1}$ (the profit associated to product manufacture is smaller) and in increase in $\phi_{3}$ (larger transition cost). This cost combination makes this production sequence worst than the first and second ones. Even thought the shape of the dynamic transitions looks also similar to the ones of first and second cases as depicted in Figure 5. 

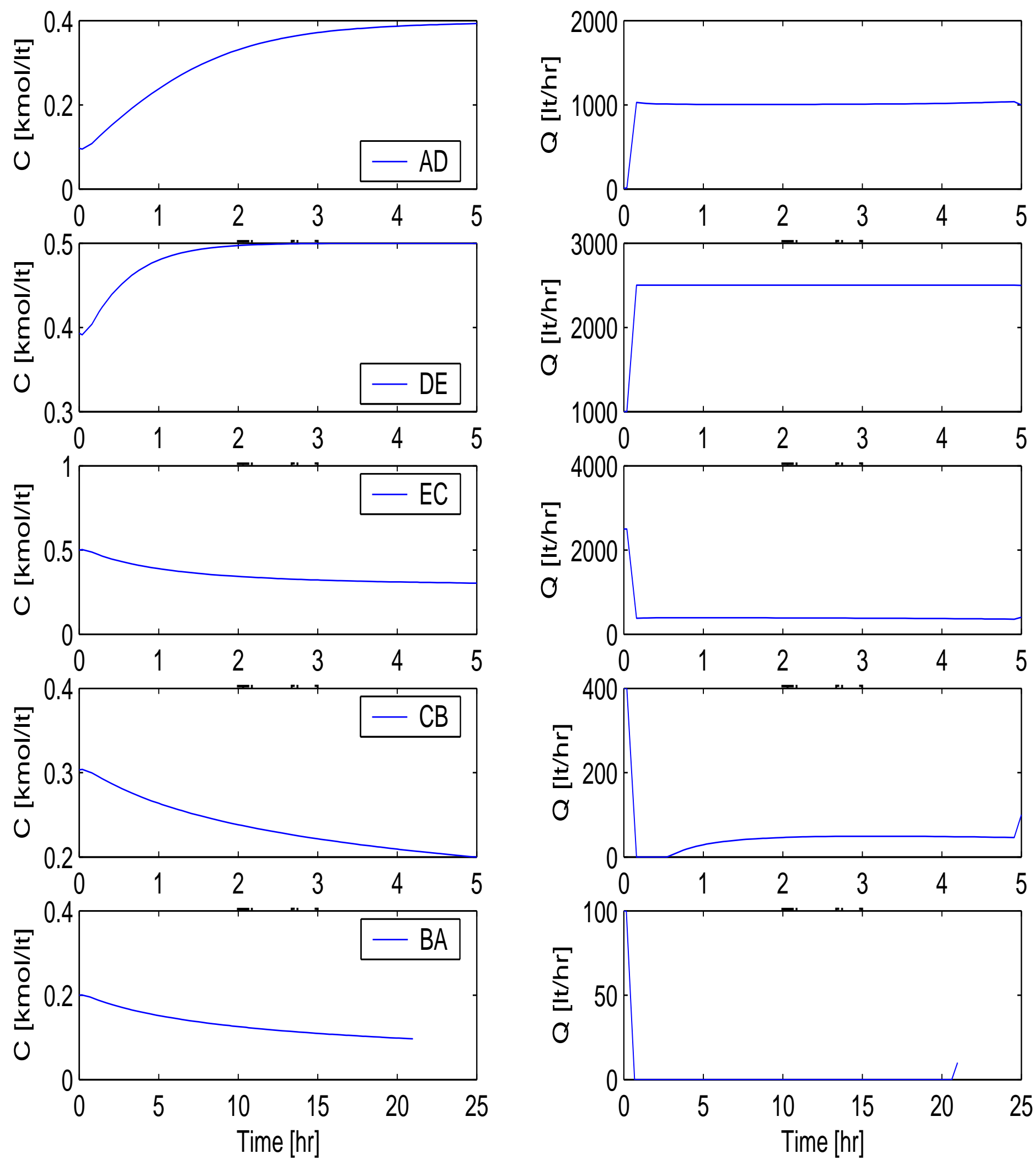

Figure 4: Optimal dynamic profiles for the volumetric flow rate and reactor concentration during product transition for the first case study, second best solution. 

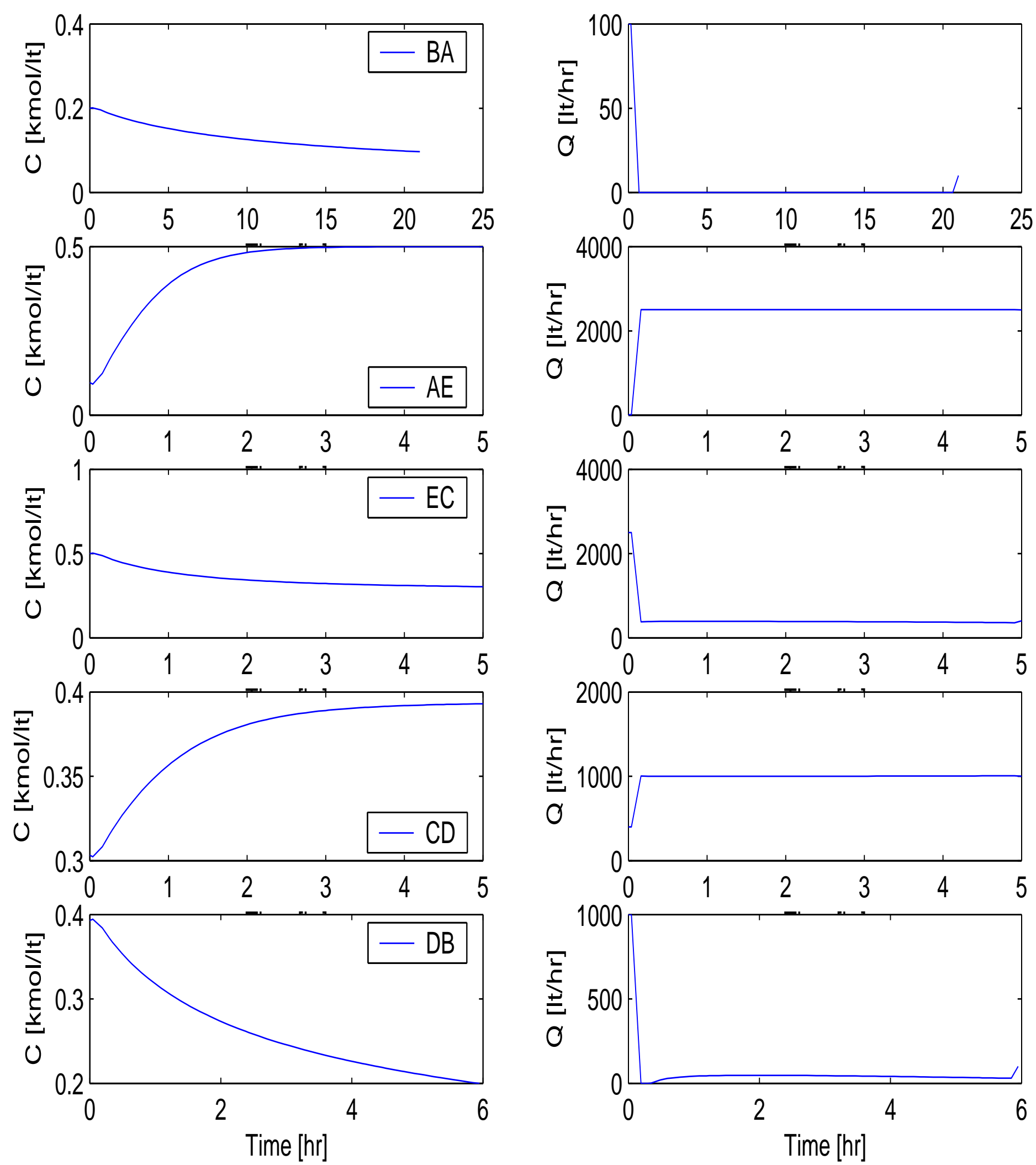

Figure 5: Optimal dynamic profiles for the volumetric flow rate and reactor concentration during product transition for the first case study, third best solution. 


\subsection{CSTR with simultaneous reactions and input multiplicities}

The following set of reactions

$$
\begin{aligned}
2 R_{1} & \stackrel{k_{1}}{\longrightarrow} A \\
R_{1}+R_{2} & \stackrel{k_{2}}{\longrightarrow} B \\
R_{1}+R_{3} & \stackrel{k_{3}}{\longrightarrow}
\end{aligned}
$$

is carried out in a continuous and isothermal stirred tank reactor displayed in Figure 6. Products $A, B$ and $C$ are manufactured using different values of the feed stream volumetric flow rates of the reactants $R_{1}, R_{2}, R_{3}$.

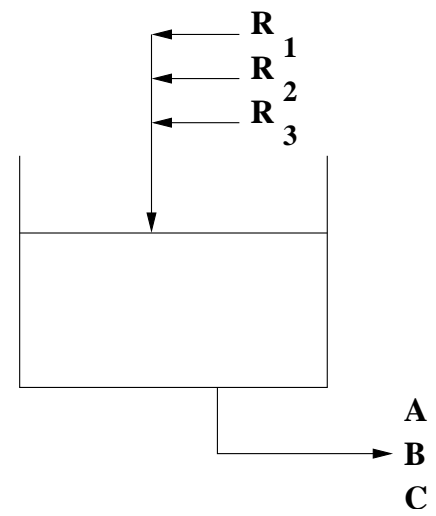

Figure 6: CSTR flowsheet for the second case study. Products $(A, B, C)$ are manufactured using different combinations of the reactants $\left(R_{1}, R_{2}, R_{3}\right)$.

The dynamic mathematical model of the above system is as follows.

$$
\begin{aligned}
\frac{d C_{R_{1}}}{d t} & =\frac{\left(Q_{R_{1}} C_{R_{1}}^{i}-Q C_{R_{1}}\right)}{V}+\mathcal{R}_{r_{1}} \\
\frac{d C_{R_{2}}}{d t} & =\frac{\left(Q_{R_{2}} C_{R_{2}}^{i}-Q C_{R_{2}}\right)}{V}+\mathcal{R}_{r_{2}} \\
\frac{d C_{R_{3}}}{d t} & =\frac{\left(Q_{R_{3}} C_{R_{3}}^{i}-Q C_{R_{3}}\right)}{V}+\mathcal{R}_{r_{3}} \\
\frac{d C_{A}}{d t} & =\frac{Q\left(C_{A}^{i}-C_{A}\right)}{V}+\mathcal{R}_{A} \\
\frac{d C_{B}}{d t} & =\frac{Q\left(C_{B}^{i}-C_{B}\right)}{V}+\mathcal{R}_{B} \\
\frac{d C_{C}}{d t} & =\frac{Q\left(C_{C}^{i}-C_{C}\right)}{V}+\mathcal{R}_{C}
\end{aligned}
$$




\begin{tabular}{||ccc||}
\hline Parameter & Value & Units \\
\hline$C_{R_{1}}^{i}$ & 1 & $\mathrm{~mol} / \mathrm{l}$ \\
$C_{R_{2}}^{i}$ & 0.8 & $\mathrm{~mol} / \mathrm{l}$ \\
$C_{R_{3}}^{i}$ & 1 & $\mathrm{~mol} / \mathrm{l}$ \\
$C_{A}^{i}$ & 0 & $\mathrm{~mol} / \mathrm{l}$ \\
$C_{B}^{i}$ & 0 & $\mathrm{~mol} / 1$ \\
$C_{C}^{i}$ & 0 & $\mathrm{~mol} / \mathrm{l}$ \\
$V$ & 6000 & $\mathrm{l}$ \\
$k_{1}$ & 0.1 & $1 /(\mathrm{min}-\mathrm{mol})$ \\
$k_{2}$ & 0.9 & $1 /(\mathrm{min}-\mathrm{mol})$ \\
$k_{3}$ & 1.5 & $1 /(\mathrm{min}-\mathrm{mol})$ \\
\hline
\end{tabular}

Table 5: Steady-state design and kinetic information for the second case study.

where the kinetic expressions follow simple mass action law kinetics,

$$
\begin{aligned}
& \mathcal{R}_{A}=k_{1} C_{R_{1}}^{2} \\
& \mathcal{R}_{B}=k_{2} C_{R_{1}} C_{R_{2}} \\
& \mathcal{R}_{C}=k_{3} C_{R_{1}} C_{R_{3}} \\
& \mathcal{R}_{r_{1}}=-\mathcal{R}_{A}-\mathcal{R}_{B}-\mathcal{R}_{C} \\
& \mathcal{R}_{r_{2}}=-\mathcal{R}_{B} \\
& \mathcal{R}_{r_{3}}=-\mathcal{R}_{C}
\end{aligned}
$$

and

$$
Q=Q_{R_{1}}+Q_{R_{2}}+Q_{R_{3}}
$$

where $Q_{R_{1}}, Q_{R_{2}}$ and $Q_{R_{3}}$ are the feed stream volumetric flow rates of reactants $R_{1}, R_{2}$ and $R_{3}$, respectively. $C^{i}$ is the reactant concentration, $C$ is the product concentration, $V$ is the reactor volume and $k$ is the kinetic rate constant. $Q$ is the total feed stream volumetricflow rate. The design and kinetic parameters are shown in Table 5. The operating conditions leading to manufacture each one of the $A, B$ and $C$ products are shown in Table 6 ; also shown are the steady-state information concerning each product. From the information contained in this table, we see that the residence time of product $A$ is larger than the corresponding ones of products $B$ and $C$. In fact product $A$ features a residence time 


\begin{tabular}{||c|ccccccccc||}
\hline Product & $Q_{R_{1}}$ & $Q_{R_{2}}$ & $Q_{R_{3}}$ & $C_{R_{1}}$ & $C_{R_{2}}$ & $C_{R_{3}}$ & $C_{A}$ & $C_{B}$ & $C_{C}$ \\
\hline$A$ & 100 & 0 & 0 & 0.333 & 0 & 0 & 0.666 & 0 & 0 \\
$B$ & 100 & 100 & 0 & 0.1335 & 0.0869 & 0 & 0.0534 & 0.3131 & 0 \\
$C$ & 100 & 0 & 100 & 0.0837 & 0 & 0.1048 & 0.021 & 0 & 0.3951 \\
\hline
\end{tabular}

Table 6: Processing conditions leading to the manufacture of the $A, B$ and $C$ products of the second case study.

\begin{tabular}{|cccc|}
\hline Product & $\begin{array}{c}\text { Demand } \\
{[\mathrm{Kg} / \mathrm{h}]}\end{array}$ & $\begin{array}{c}\text { Product } \\
\text { cost }[\$ / \mathrm{kg}]\end{array}$ & $\begin{array}{c}\text { Inventory } \\
\text { cost }[\$]\end{array}$ \\
\hline$A$ & 5 & 500 & 1 \\
$B$ & 10 & 400 & 1.5 \\
$C$ & 15 & 600 & 1.8 \\
\hline
\end{tabular}

Table 7: Demand rate and product and inventory costs for the second case study reaction system.

value that is exactly the double of products $B$ and $C$ residence times. This indicates that transition from any product to product $A$ will be slower that from product $A$ to any other product. This observation will be verified later by computing the open-loop poles of each product.

In reaction systems featuring intermediate products, as the problem at hand, there is the risk of input multiplicities. Such kind of nonlinear behavior creates a situation where the same state value is obtained for two different values of the manipulated variable. From a closed-loop control point this behavior is undesirable since, under certain conditions, it has been related to the presence of right-hand plane zeros [18], which limit the response speed of the closed-loop system. The emergence of right-hand plane zeros makes the use of PID controllers impractical because of slope sign changes [19]. Until now the only way of dealing with input multiplicities has been to use a controller able to deal with those systems or by system redesign. In our case, for the operating and processing conditions shown Tables 5 and 6 , the reaction system displays input multiplicities as shown in Figure 7. Input multiplicities were only found for the $B$ and $C$ products; monotonic behavior was always observed for the $A$ product.

Information regarding the demand rate, inventory and product costs is shown in Table 


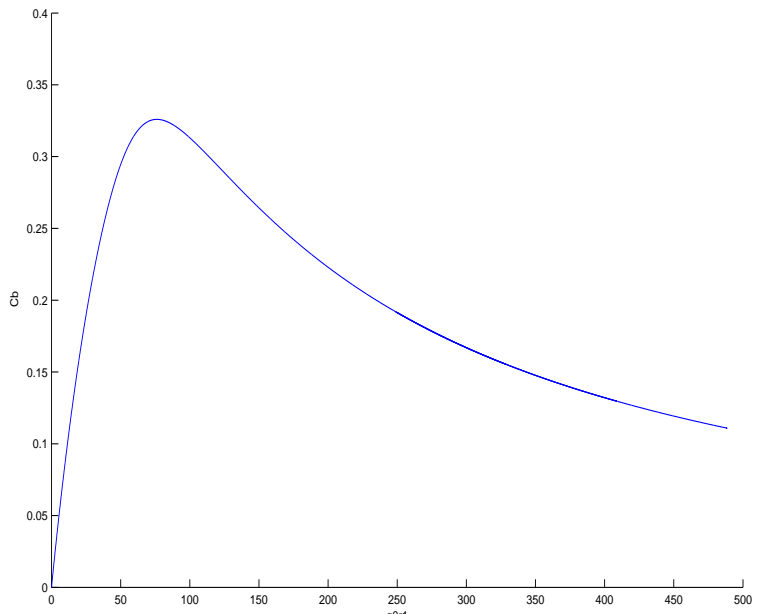

(a)

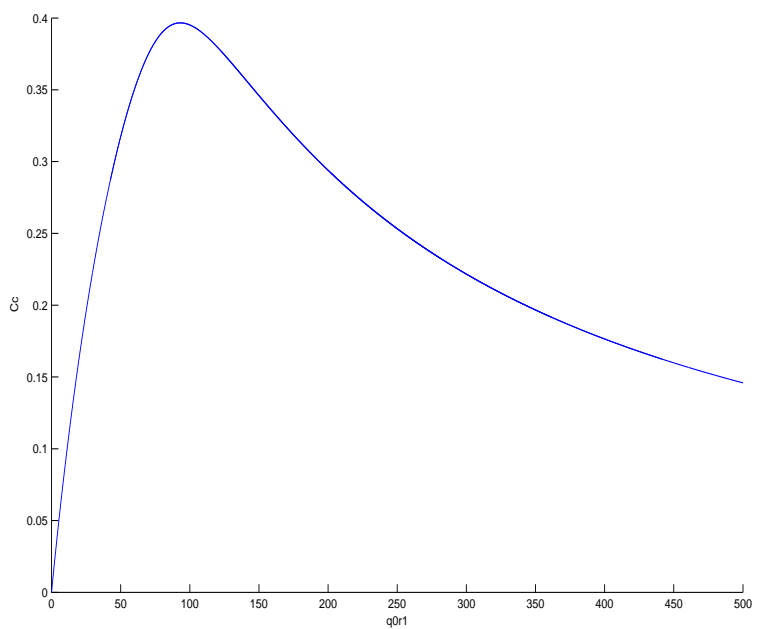

(c)

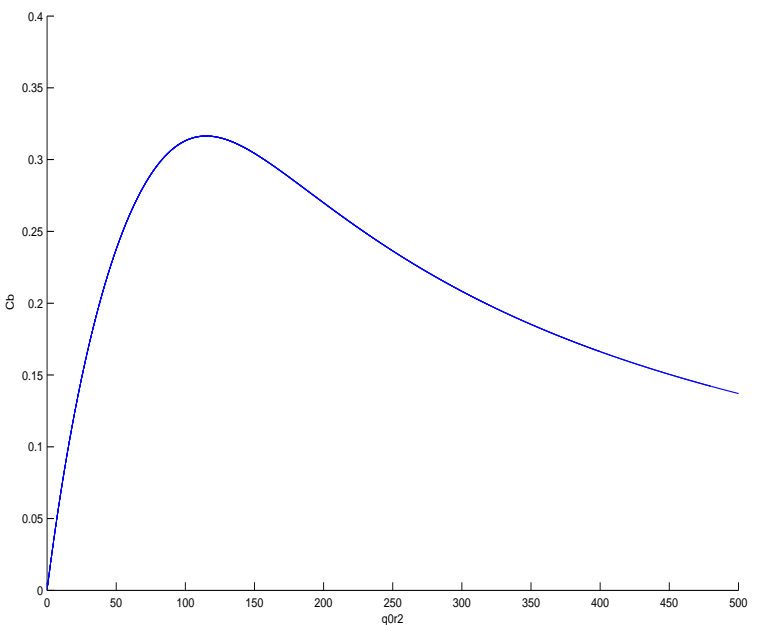

(b)

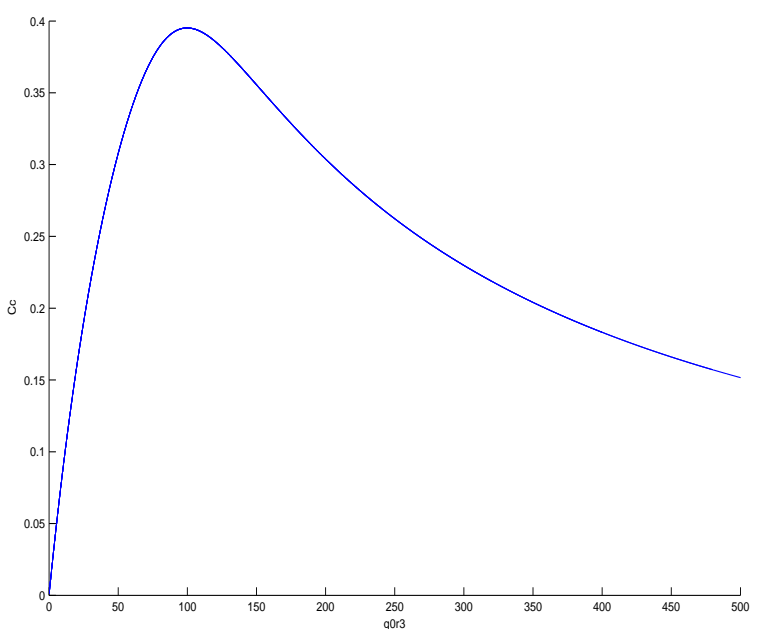

(d)

Figure 7: Input multiplicities in the second case study. (a) and (b) refer to product B using $Q_{R_{1}}^{o}$ and $Q_{R_{2}}^{o}$ as continuation parameters, respectively. Similarly, (c) and (d) refer to product $\mathrm{C}$ using $Q_{R_{1}}^{o}$ and $Q_{R_{3}}^{o}$ as continuation parameters, respectively, while $C_{b}$ and $C_{c}$ stand for composition of products $B$ and $C$, respectively.

\begin{tabular}{|cccccccc|}
\hline Slot & Product & $\begin{array}{c}\text { Process time } \\
{[\mathrm{m}]}\end{array}$ & $\begin{array}{c}\text { Production rate } \\
{[\mathrm{Kg} / \mathrm{m}]}\end{array}$ & $\begin{array}{c}w \\
{[\mathrm{Kg}]}\end{array}$ & $\begin{array}{c}\text { Transition Time } \\
{[\mathrm{m}]}\end{array}$ & $\begin{array}{c}\text { T start } \\
{[\mathrm{m}]}\end{array}$ & $\begin{array}{c}\text { T end } \\
{[\mathrm{m}]}\end{array}$ \\
\hline 1 & $C$ & 204.2 & 89.52 & 18273.3 & 15 & 0 & 219.2 \\
2 & $B$ & 44.5 & 71.31 & 3174.4 & 15 & 219.2 & 278.7 \\
3 & $A$ & 23.8 & 66.7 & 1587.2 & 15 & 278.7 & 317.5 \\
\hline
\end{tabular}

Table 8: Simultaneous scheduling and control results for the second case study. The objective function value is $\$ 32388$ and 317.5 of total cycle time. 


\begin{tabular}{|cccc|}
\hline Pole & \multicolumn{3}{c|}{ Product } \\
& A & B & C \\
\hline 1 & -0.0167 & -0.0333 & -0.0333 \\
2 & -0.0167 & -0.0333 & -0.0333 \\
3 & -0.0167 & -0.0333 & -0.0333 \\
4 & -0.0833 & -0.0486 & -0.3258 \\
5 & -0.5167 & -0.2431 & -0.0405 \\
6 & -0.3167 & -0.2335 & -0.1087 \\
\hline
\end{tabular}

Table 9: Open-loop poles for products $A, B$ and $C$ of the second case study.

7, while simultaneous optimal scheduling and control results are shown in Table 8. As shown there, the optimizer selected the cyclic $C \rightarrow B \rightarrow A$ production sequence. Figure 8 depicts the optimal state transitions for this production sequence. From information contained in Table 8, it is clear that the optimizer selects first to produce as much as possible of product $C$ because it is the most valuable product. Next, the optimizer decides to switch to manufacture product $B$ because transition time from product $C$ to product $B$ is shorter than to product $A$. The points raised about the speed of response between products can be explained by looking at the open-loop poles of each one product shown in Table 9 . From this table it is interesting to note that products $B$ and $C$ share the same poles and therefore they feature the same time constant. The dominant pole of product $A$ is located closer to the imaginary axis, an indication that it has a larger time constant and a slower speed of response. This partially helps to explain the reason why the optimizer selected the cyclic $C \rightarrow B \rightarrow A$ production sequence as the optimal one. 

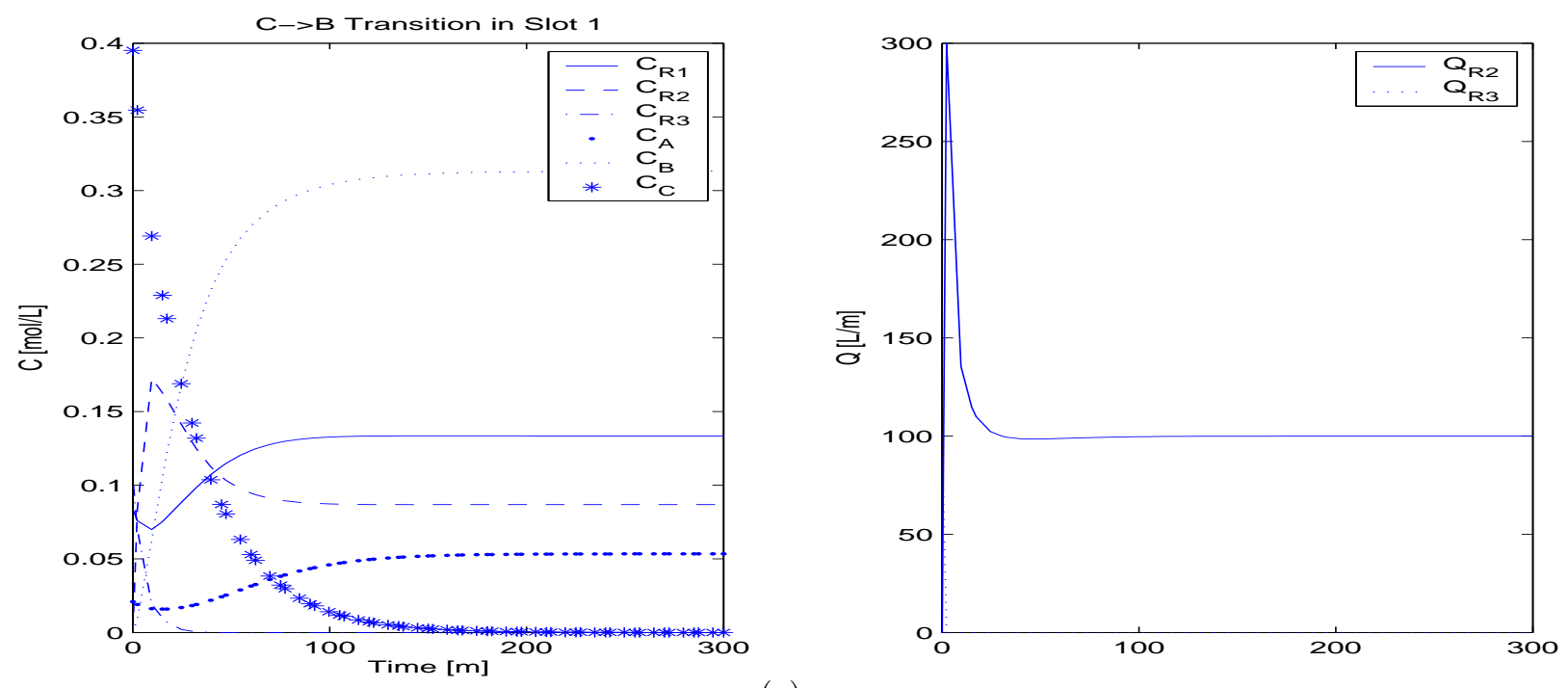

(a)
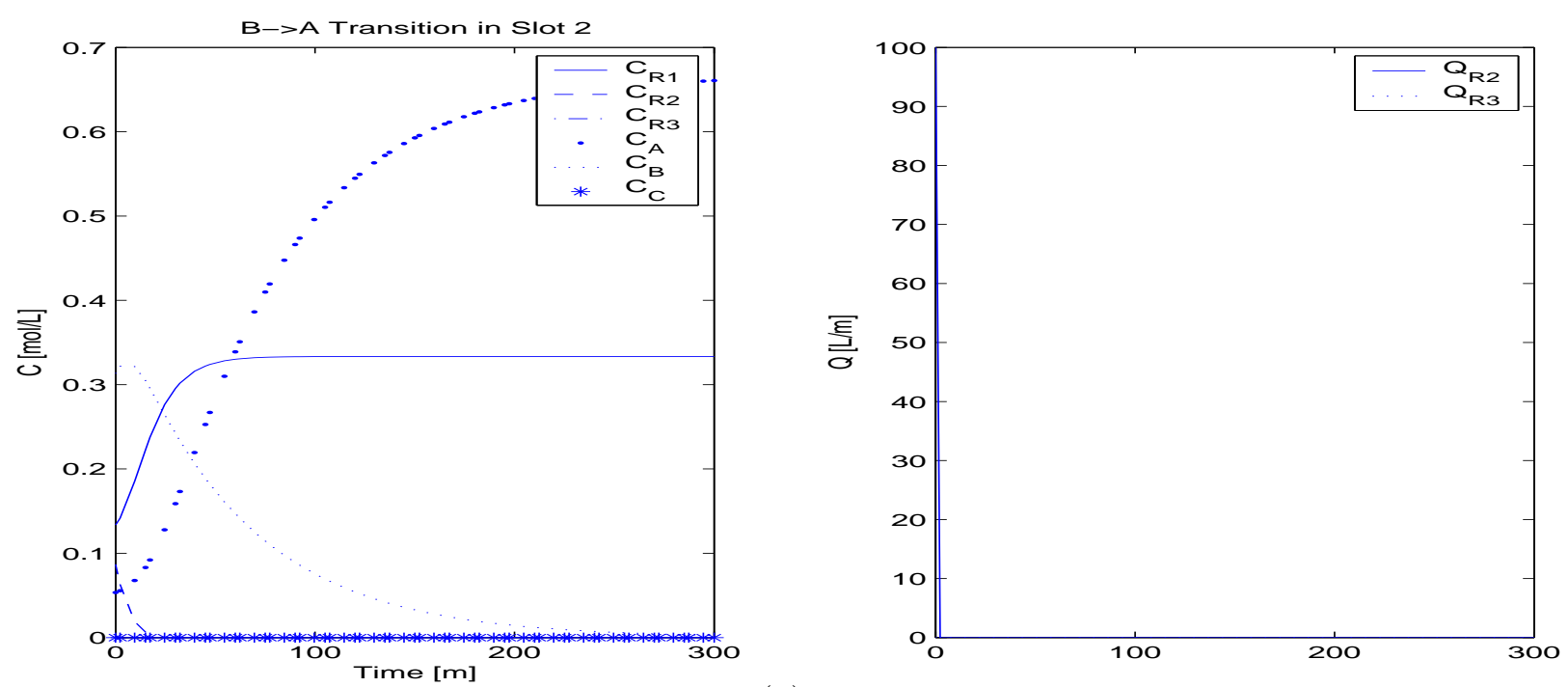

(b)
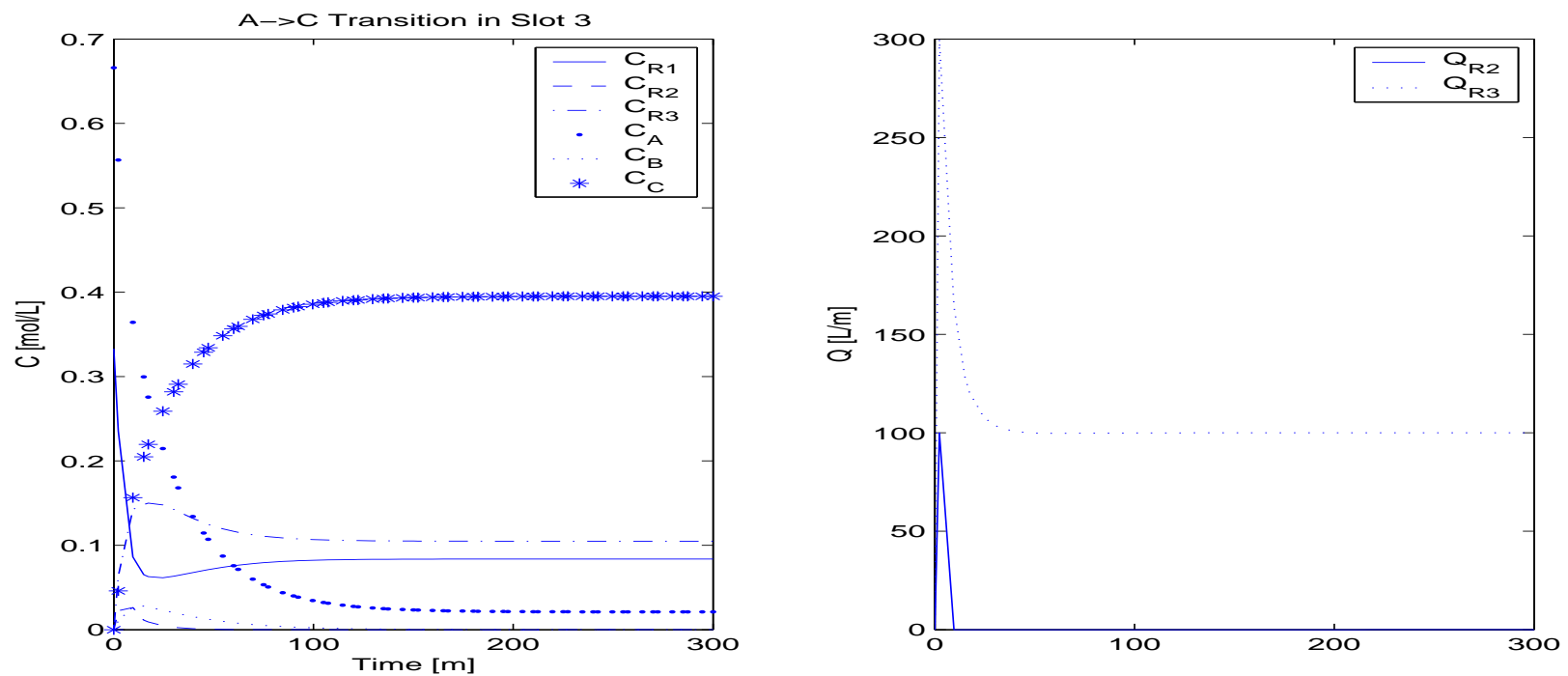

(c)

Figure 8: Optimal schedule and dynamic profiles for reactor concentrations and volumetric feed flow rates for the second example. 
Similarly to the first case of study, we tried to identify a second best optimal production sequence solution. After some trials, the optimizer selected the cyclic $A \rightarrow C \rightarrow B$ production sequence as another optimal one. The first and second solutions feature the same objective function value, production times, process rates, etc as shown in Table 8 for the first production sequence, being the sequence in which products are manufactured the only difference between both production sequences. The reason why both production sequences feature the same values of the decision variables is due to the fact that the production sequence is cyclic and the time horizon is infinite. Another reason has to do with the fact that, for the processing conditions shown in Tables 5 and 6 , the transition costs turn out to be small. This means that the resulting production sequence will be almost independent of the transition dynamics and it will be most influenced by the cost of the products and inventories. Anyway, it is interesting to remark that also in this case the optimizer always select first the product transitions that feature easy to carry out product transitions, leaving at the end the transition from the farthest to the closest to the imaginary axis pole. Figure 9 depicts the dynamic optimal transition trajectories for the second optimal production sequence. Analogously to the steady-state optimal results, the dynamic optimal transition trajectories of the second and first solutions are the same. 

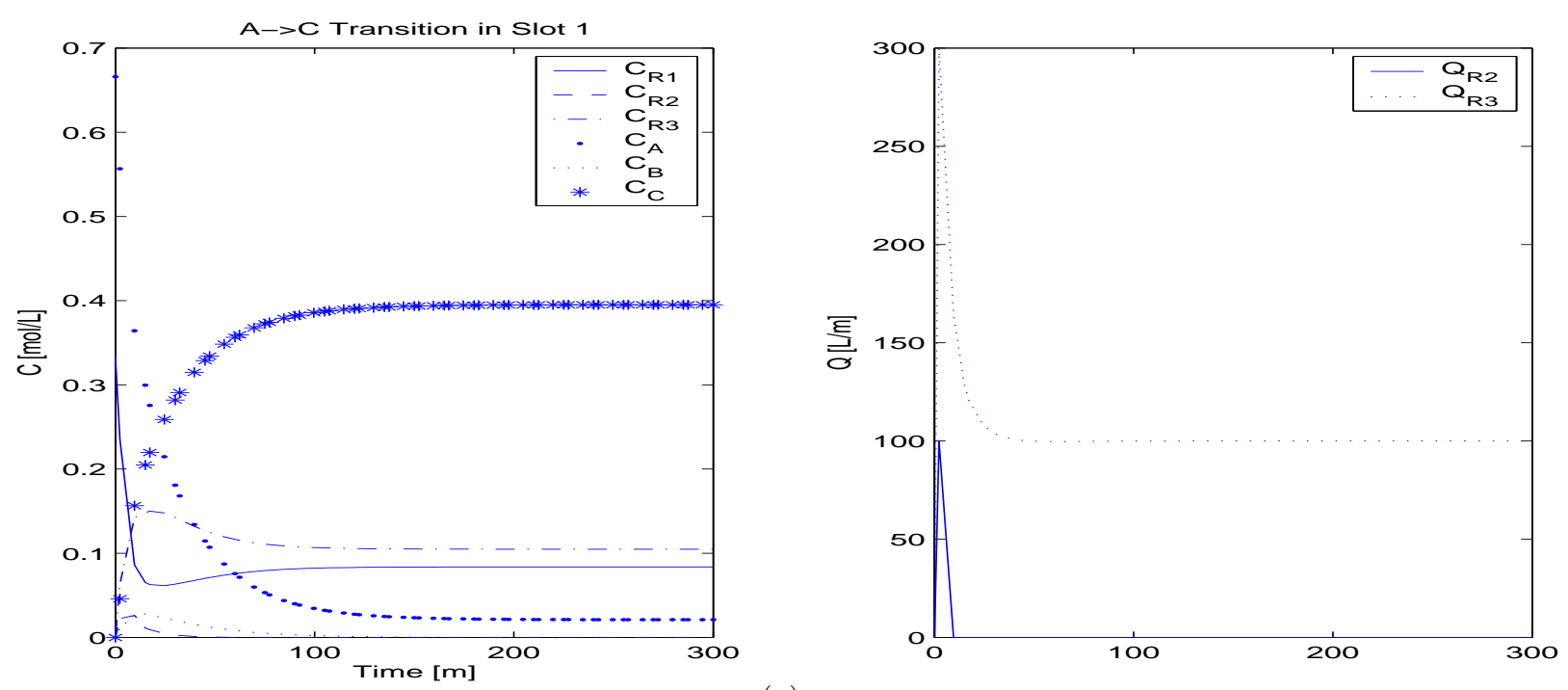

(a)
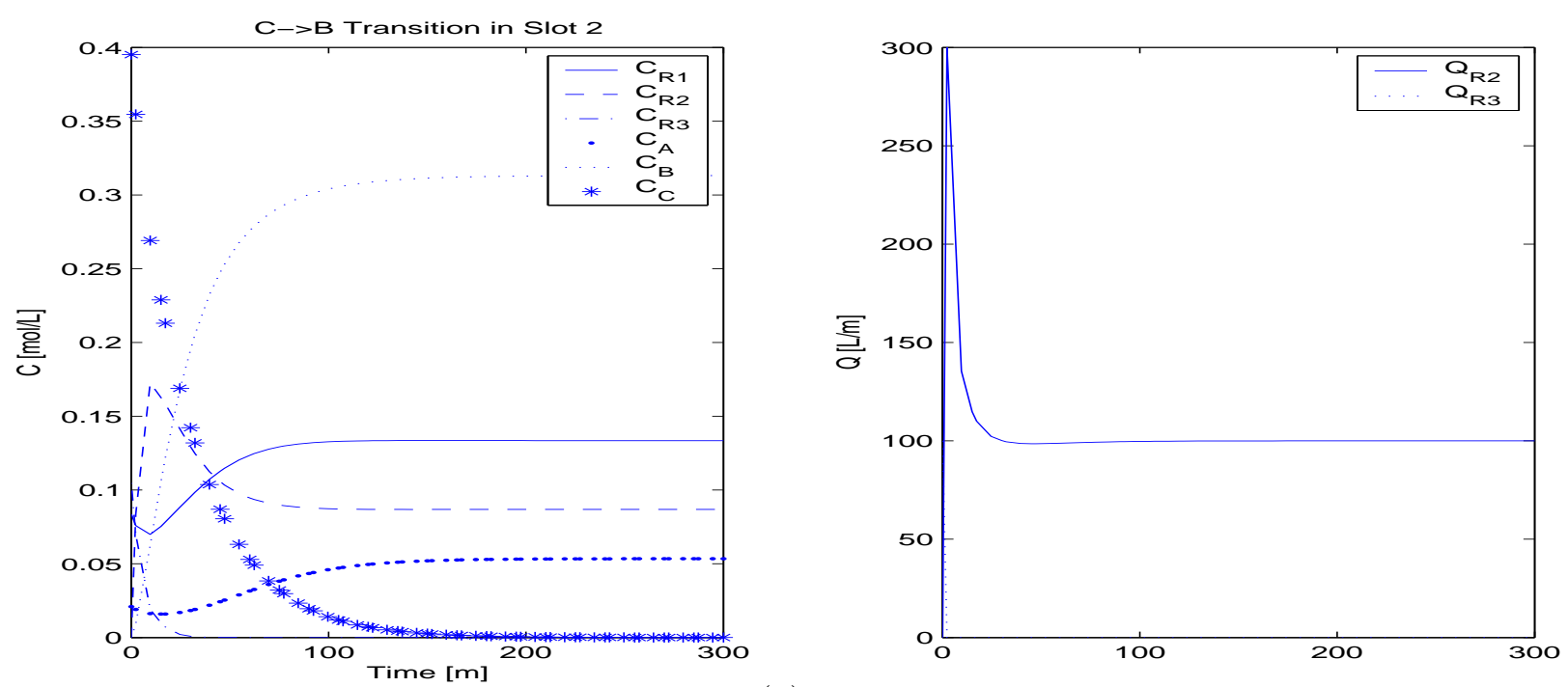

(b)
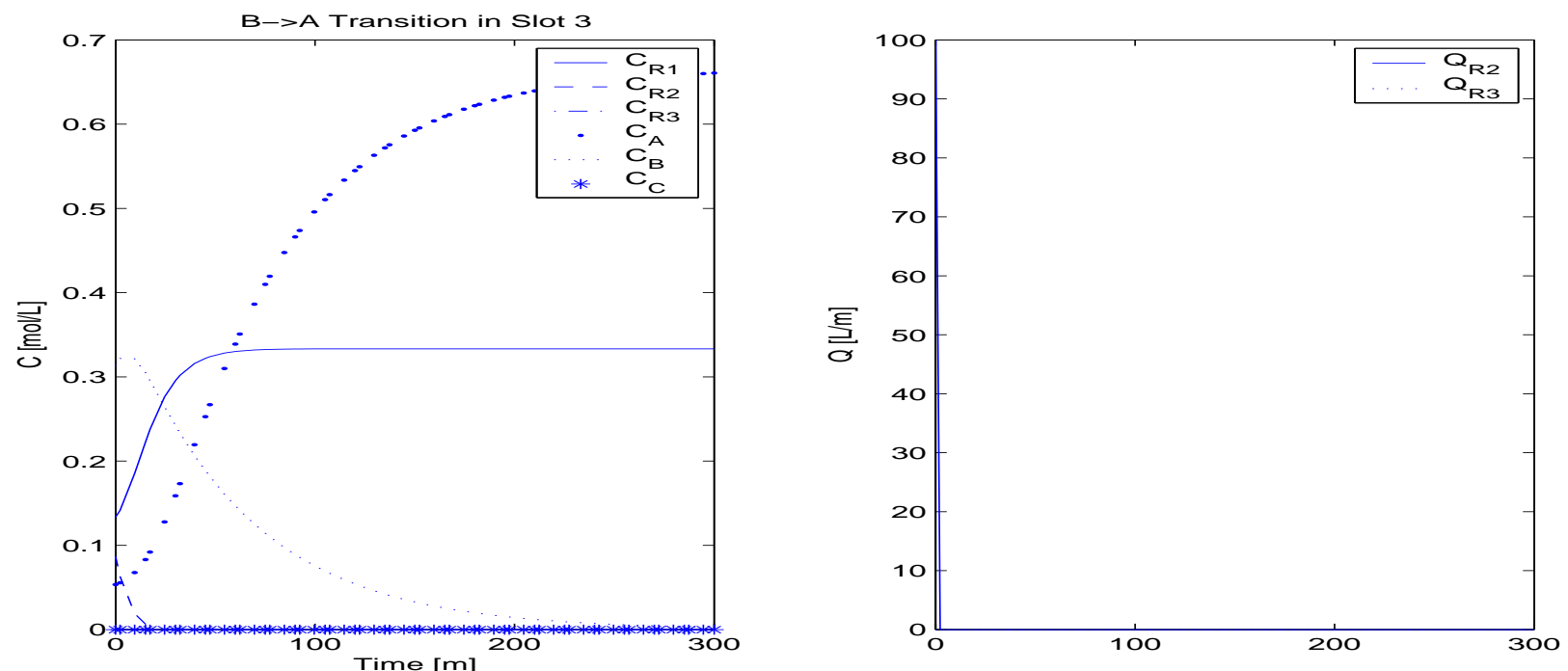

(c)

Figure 9: Optimal schedule and dynamic profiles for reactor concentrations and volumetric feed flow rates for the second example, second solution. 


\subsection{CSTR with output multiplicities}

In order to compute dynamic optimal transition trajectories around highly nonlinear regions, the CSTR model as proposed by Hicks and Ray [20] was used. Because the original parameters set used by these authors did not lead to multiple steady-states, some of the values were modified in order to end-up with a multiplicity map. In dimensionless form the model is given by:

$$
\begin{aligned}
\frac{d y_{1}}{d t} & =\frac{1-y_{1}}{\theta}-k_{10} e^{-N / y_{2}} y_{1} \\
\frac{d y_{2}}{d t} & =\frac{y_{f}-y_{2}}{\theta}+k_{10} e^{-N / y_{2}} y_{1}-\alpha u\left(y_{2}-y_{c}\right)
\end{aligned}
$$

where $y_{1}$ stands for dimensionless concentration $\left(c / c_{f}\right), y_{2}$ is the dimensionless temperature $\left(T / J c_{f}\right), y_{c}$ is the dimensionless coolant temperature $\left(T_{c} / J c_{f}\right), y_{f}$ is the dimensionless feed temperature $\left(T_{f} / J c_{f}\right)$, and $u$ is the cooling flowrate. Table 10 contains the numerical values of the parameters used in this work; this set of parameter values lead to operate around the multiplicity region shown in Figure 10.

Table 10: Parameters values for the third case study featuring output nonlineaties.

\begin{tabular}{|ccl|ccl|}
\hline$\theta$ & 20 & Residence time & $T_{f}$ & 300 & Feed temperature \\
$J$ & 100 & $(-\Delta H) /\left(\rho C_{p}\right)$ & $k_{10}$ & 300 & Preexponential factor \\
$c_{f}$ & 7.6 & Feed concentration & $T_{c}$ & 290 & Coolant temperature \\
$\alpha$ & $1.95 \times 10^{-4}$ & Dimensionless heat transfer area & $N$ & 5 & $E_{1} /\left(R J_{c_{f}}\right)$ \\
\hline
\end{tabular}

Our goal is to manufacture four products denoted as $A, B, C$ and $D$. Operating condi-

\begin{tabular}{|cccc|}
\hline Product & $\begin{array}{c}\text { Demand } \\
{[\mathrm{Kg} / \mathrm{h}]}\end{array}$ & $\begin{array}{c}\text { Product } \\
\text { cost }[\$ / \mathrm{kg}]\end{array}$ & $\begin{array}{c}\text { Inventory } \\
\text { cost }[\$]\end{array}$ \\
\hline$A$ & 100 & 100 & 1 \\
$B$ & 200 & 50 & 1.3 \\
$C$ & 400 & 30 & 1.4 \\
$D$ & 500 & 80 & 1.1 \\
\hline
\end{tabular}

Table 11: Process data for the third case study. $A, B, C$ and $D$ stand for the four products to be manufactured. Information about the steady-state design for each one of the products is shown in Figure 10. 


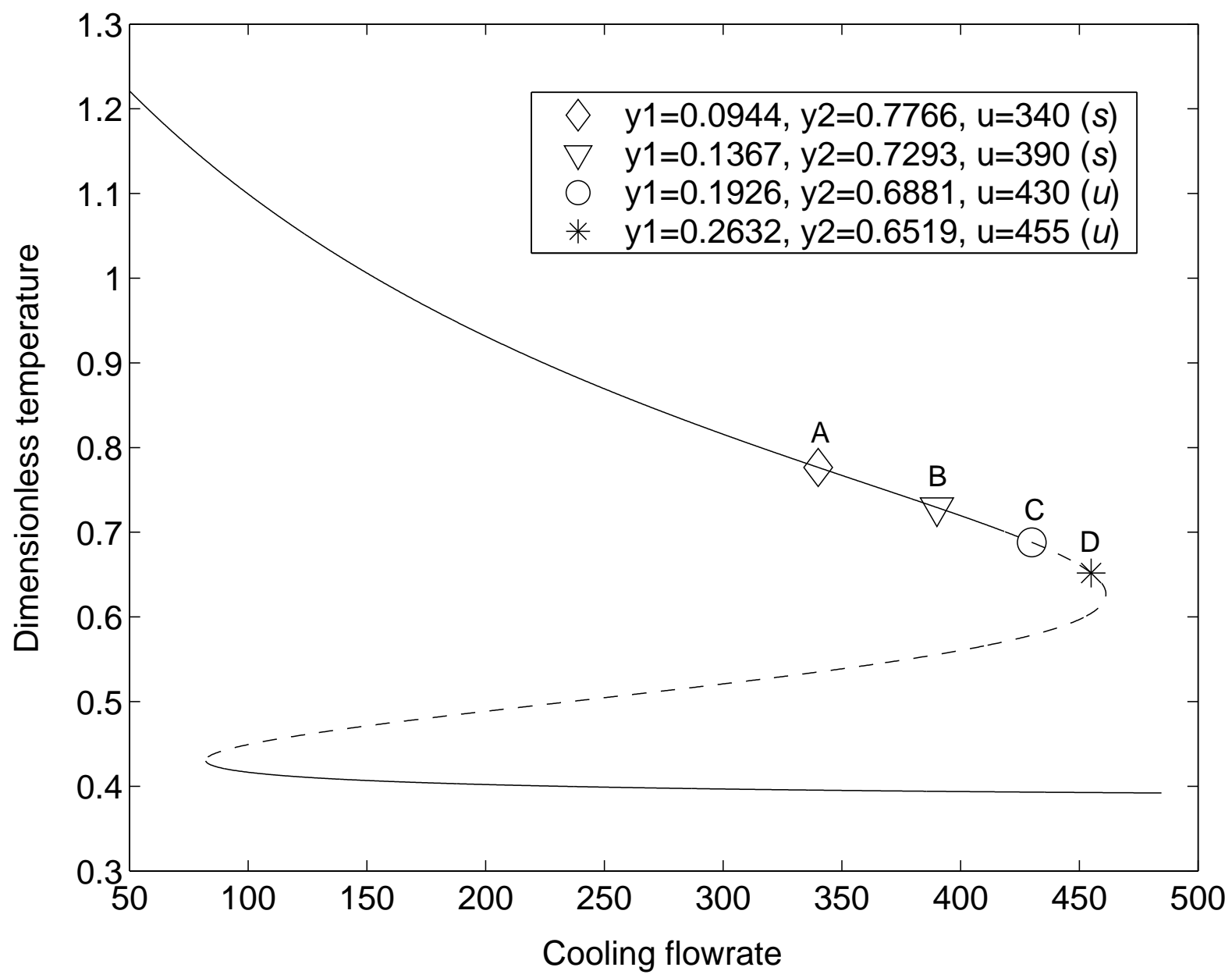

Figure 10: Multiplicity map (— stable solution, -- unstable solution).

tions are also displayed in Figure 10. Note that the $A$ and $B$ products are manufactured around open-loop stable steady-states. The $C$ operating point is located at the point where a stability interchange, together with a Hopf bifurcation point, takes place. Finally, the $D$ product is manufactured around a completely unstable open-loop operating region. In all the cases the manipulated variable is the cooling flow rate $u$. One of the aims of this case study is to demonstrate that, even in the face of highly non-linear operating regions and open-loop unstable systems, our proposed simultaneous scheduling and control formulation is able to perform satisfactorily and to determine an optimal scheduling and control solution. Information regarding the production rate, demand rate and inventory costs is shown in Table 11 .

In this case, the $A \rightarrow B \rightarrow C \rightarrow D$ scheduling turned out to be the optimal cyclic 


\begin{tabular}{|cccccccc|}
\hline Slot & Product & $\begin{array}{c}\text { Process time } \\
{[\mathrm{h}]}\end{array}$ & $\begin{array}{c}\text { Production rate } \\
{[\mathrm{Kg} / \mathrm{h}]}\end{array}$ & $\begin{array}{c}w \\
{[\mathrm{Kg}]}\end{array}$ & $\begin{array}{c}\text { Transition Time } \\
{[\mathrm{h}]}\end{array}$ & $\begin{array}{c}\text { T start } \\
{[\mathrm{h}]}\end{array}$ & $\begin{array}{c}\text { T end } \\
{[\mathrm{h}]}\end{array}$ \\
\hline 1 & $A$ & 28.3 & 559.9 & 15831.7 & 10 & 0 & 38.3 \\
2 & $B$ & 13.1 & 613.6 & 8044.9 & 10 & 38.3 & 61.4 \\
3 & $C$ & 13.4 & 656.1 & 8748.9 & 10 & 61.4 & 84.8 \\
4 & $D$ & 5.8 & 688.3 & 4022.5 & 10 & 84.8 & 100.6 \\
\hline
\end{tabular}

Table 12: Simultaneous scheduling and control results for the third case study. The objective function value is $\$ 7657$ and $100.6 \mathrm{~h}$ of total cycle time.

production sequence. The profit is $\$ 7657$ with a total cycle time of $100.6 \mathrm{hr}$. The rest of the decision variables optimal values are shown in Table 12. It is again interesting to notice that the optimizer decides first to manufacture as much as possible of the most valuable product $A$. After that, the optimizer selected product transitions that are faster, in the sense that the pole of each succesive product is closer to the imaginary exis (except for product $D$ ). The open-loop poles of each product are shown in Table 13. It should be remarked that, in this case study, we have two products $(C, D)$ whose manufacture demands to operate around open-loop unstable operating points. The computation of open-loop dynamic optimal trajectories for open-loop unstable systems is difficult to carry out using dynamic optimization strategies based upon the so-called sequential approach [21]. On the other hand, the simultaneous approach [22] efficiently and naturally deals with this type of problems without using tricks such closed-loop stabilization of the originally unstable system and then running the dynamic optimization problem. In a previous work [22] we have provided some theoretical explainations why the simultaneous approach copes with open-loop unstable systems. Not surprisingly the minimum transition time, independently of the type of transition, is $10 \mathrm{hr}$. This results is in agreement with previous calculations related to the open-loop dynamic optimization of the same reaction system [23]. Another point to stress is that our SSC formulation is able to cope with product transitions between highly nonlinear regions. As a matter of fact, the $B \rightarrow C$ transitions involves transition from a open-loop stable to an unstable system through a Hopf bifurcation point where oscillatory behavior emerges.

From the open-loop poles shown in Table 13, we should expect to have oscillatory 


\begin{tabular}{|ccccc|}
\hline Pole & \multicolumn{4}{c|}{ Product } \\
& A & B & C & D \\
\hline 1 & $-0.1352+0.1566 \mathrm{i}$ & $-0.0430+0.1548 \mathrm{i}$ & $0.0164+0.1147 \mathrm{i}$ & $0.0524+0.0440 \mathrm{i}$ \\
2 & $-0.1352-0.1566 \mathrm{i}$ & $-0.0430-0.1548 \mathrm{i}$ & $0.0164-0.1147 \mathrm{i}$ & $0.0524-0.0440 \mathrm{i}$ \\
\hline
\end{tabular}

Table 13: Open-loop poles for products $A, B, C$ and $D$ of the third case study.

\begin{tabular}{|cccccccc|}
\hline Slot & Product & $\begin{array}{c}\text { Process time } \\
{[\mathrm{h}]}\end{array}$ & $\begin{array}{c}\text { Production rate } \\
{[\mathrm{Kg} / \mathrm{h}]}\end{array}$ & $\begin{array}{c}w \\
{[\mathrm{Kg}]}\end{array}$ & $\begin{array}{c}\text { Transition Time } \\
{[\mathrm{h}]}\end{array}$ & $\begin{array}{c}\text { T start } \\
{[\mathrm{h}]}\end{array}$ & $\begin{array}{c}\text { T end } \\
{[\mathrm{h}]}\end{array}$ \\
\hline 1 & $D$ & 6.07 & 559.9 & 4176.7 & 10 & 0 & 16.07 \\
2 & $A$ & 28.9 & 613.6 & 16177.2 & 10 & 16.07 & 55 \\
3 & $C$ & 13.9 & 656.1 & 9084.3 & 12 & 55 & 80.8 \\
4 & $B$ & 13.7 & 688.3 & 8353.4 & 10 & 80.8 & 104.4 \\
\hline
\end{tabular}

Table 14: Simultaneous scheduling and control results for the third case study, second solution. The objective function value is $\$ 6070.6$ and $104.4 \mathrm{~h}$ of total cycle time.

response for product transitions because most of the poles have nonzero imaginary part. Product transitions ending at product $D$ should feature the weakest oscillatory behavior because its imaginary part is rather small. These observations are supported looking at the dynamic profiles of both the manipulated and controlled variables as depicted in Figure 11. Moreover, we would like to highlight the fact that the nonlinear nature of the product transitions addressed in this case study can also be appreciated in the dynamic behavior of the manipulated variable $u$. In the previous two case studies, the manipulated variable(s) always featured a simple step-like form mainly due to the presence of mild nonlinearities embedded into the system. However, in the present case study the dynamic optimal shape of the manipulated variable is not obvious. This is particularly true for the $B \rightarrow C$ and for the $C \rightarrow D$ product transitions that are placed deeper into the unstable region.

For comparison purposes of the present solution, we found a second suboptimal solution featuring the cyclic $D \rightarrow A \rightarrow C \rightarrow B$ production sequence. In this case, the objective function value was 6070.6 and 104.4 of total cyclic time. The rest of the optimal values of the decision variables are shown in Table 14. This solution is suboptimal because the optimizer decided first to manufacture product $D$ and from there to carry out the product transition for starting the manufacture of product $A$. This product tran- 

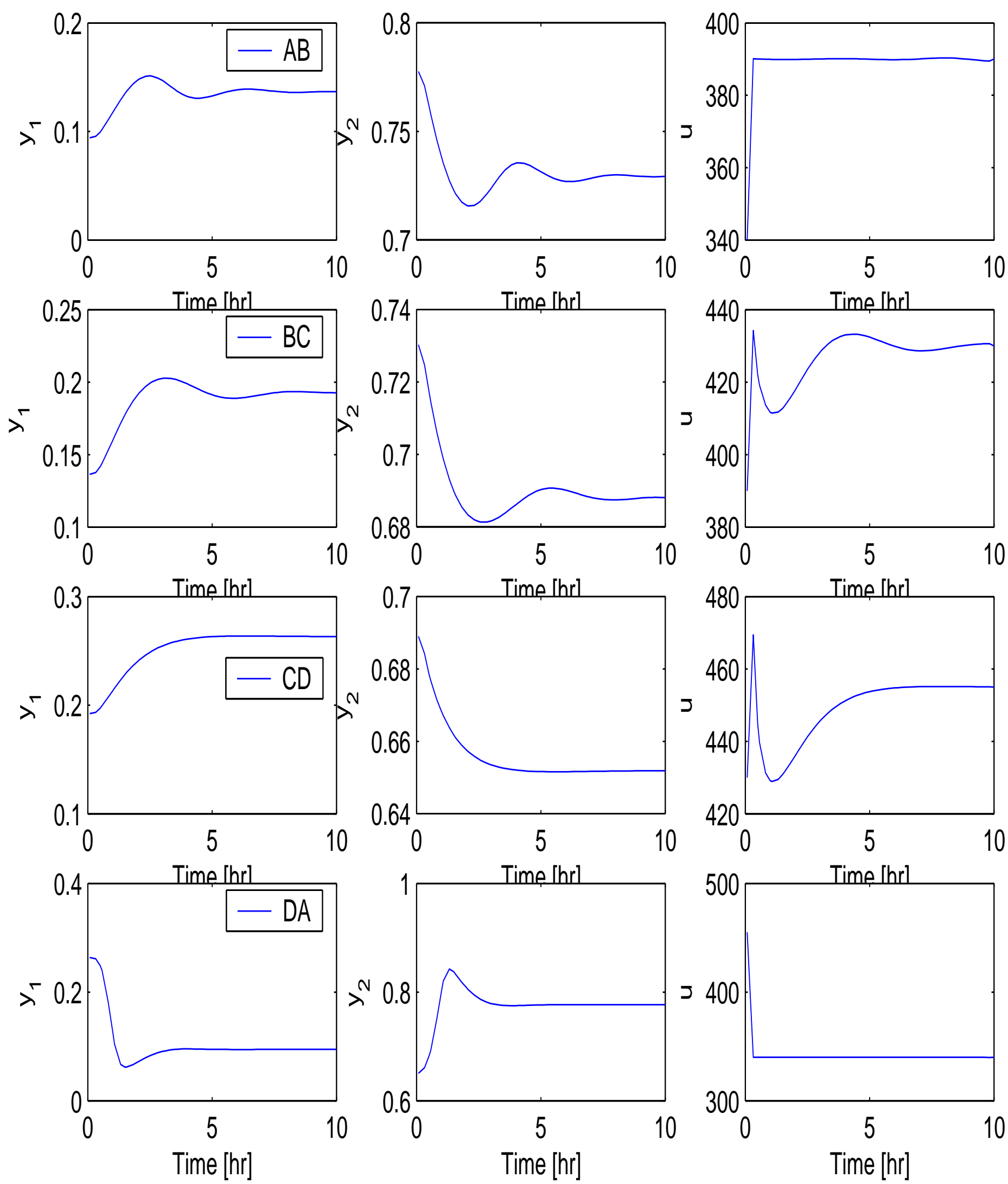

Figure 11: Optimal schedule and dynamic profiles for the third case study. 
sition turns out to be more expensive because it is hard to perform since it involves the widest variation in the open-loop pole location. From there, the optimizer keeps making product transitions that are not the best ones in terms of the transition costs. From product $A$ the optimizer selects to carry out a transition towards product $C$ and from here to product $B$. Those product transitions are definitely not the best ones in terms of transition costs. To support the past statements, we computed the $\phi_{1}, \phi_{2}$ and $\phi_{3}$ terms, as defined in Equations 27-29, of the objective function of the present case study. For the first optimal solution these values turn out to be $\left[\phi_{1}, \phi_{2}, \phi_{3}\right]=[25553.2,17633.8,263]$, while for the second suboptimal solution those values are [25303, 18252,981], in the same order. From this comparison, we can see that the profit associated with product manufacture and inventory costs are similar for both production sequences. The main difference lies in the transition costs. This explains why the cyclic $D \rightarrow A \rightarrow C \rightarrow B$ production sequence is a suboptimal solution. The dynamic profiles of both systems states and the manipulated variable are depicted in Figure 12. Again, the nonlinear behavior embedded into the present system can be seen in the shape of the manipulated variable between product transitions.

\section{Conclusions}

In this work we addressed the simultaneous cyclic scheduling and control problem for several multiproduct CSTRs. Rather than assuming constant transition times and neglecting process dynamics, a mathematical model, able to describe dynamic process behavior during product transition, was embedded into the optimization formulation. Solving the scheduling and control problem taking into account process dynamics is the rigorous way to address scheduling problems. There is always a risk of getting suboptimal solutions when process dynamics are neglected.

Because highly optimized chemical processes tend to operate around nonlinear operating regions, we selected as case studies three problems involving reaction systems, two of which display highly nonlinear behavior in the form of input and output multiplicities, 
Hopf bifurcation points and open-loop unstable operating regions. Even in face of nonlinear behavior, the proposed simultaneous cyclic scheduling and control formulation was able to find optimal production sequences. However, it was observed that convergence towards the optimal solution turned out to be harder to achieve as the nonlinearity of the system increased. Moreover, the presence of nonlinearities creates nonconvexities in the optimization formulation probably leading to obtain suboptimal solutions.

From the results obtained in this work, we think that when dealing with larger dimension systems featuring stronger nonlinear behavior, MIDO formulations, like the one presented, need to be improved to cope with such complex dynamic systems. In our opinion, presently the direct solution of MIDO problems for systems with the above mentioned characteristics does not look feasible and it might require excessive CPU time. Therefore, a decomposition strategy that exploits the natural structure of scheduling and control MIDO problems, need to be developed. Other interesting extensions of the present work consists of the case when several reactors operate in parallel [24] and in the simultaneous optimization of planning, scheduling and control [25], [26]. 


\section{References}

[1] C.A. Mendéz, J. Cerda, I.E. Grossmann, I. Harjunkoski, and M. Fahl. State of the Art Review of Optimization Methods for Short-Term Scheduling of Batch Processes. Submitted for publication, 2005.

[2] L.T. Biegler. Optimization strategies for complex process models. Advances in Chemical Engineering, 18:197-256, 1992.

[3] T. Bhatia and L.T. Biegler. Dynamic Optimization in the Design and Scheduling of Multiproduct Batch Plants. Ind. Eng. Chem. Res., 35:2234-2246, 1996.

[4] R. Mahadevan, F.J. Doyle, and A.C. Allcock. Control-Relevant Scheduling of Polymer Grade Transitions. AICHE J., 48(8):1754-1764, 2002.

[5] D. Feather, D. Harrell, R. Lieberman, and F.J. Doyle. Hybrid Approach to Polymer Grade Transition Control. AICHE J., 50(10):2502-2513, 2004.

[6] B. V. Mishra, E. Mayer, J. Raisch, and A. Kienle. Short-Term Scheduling of Batch Processes. A Comparative Study of Differentes Approaches. Ind. Eng. Chem. Res., 44:4022-4034, 2005.

[7] R. H. Nystrom, R. Franke, I. Harjunkiski, and A. Kroll. Production Campaing Planning Including Grade Transition Sequencing and Dynamic Optimization. Comput. Chem. Eng., 29(10):2163-2179, 2005.

[8] C. Chatzidoukas, C. Kiparissidis, J.D. Perkins, and Pistikopoulos E.N. Optimal Grade Transition Campaign Scheduling in a Gas Phase Polyolefin FBR Using Mixed Integer Dynamic Optimization. Process System Engineering, pages 744-747. Elsevier, 2003.

[9] R. J. Allgor and P. Barton. Mixed-Integer Dynamic Optimization I: Problem Formulation. Comput. Chem. Eng., 23(4-5):567-584, 1999. 
[10] P. Smania and J.M. Pinto. Mixed Integer Nonlinear Programming Techniques for the Short Term Scheduling of Oil Refineries. Process System Engineering, pages 744-747. Elsevier, 2003.

[11] A. Flores-Tlacuahuac and Lorenz T. Biegler. Simultaneous Mixed-Integer Dynamic Optimization for Integrated Design and Control. Manuscript in revision (Computers and Chemical Engineering), 2005.

[12] M.A. Duran and I.E. Grossmann. An outer approximation algorithm for a class of mixed integer nonlinear programs. Math.Prog., 36:307, 1986.

[13] J. Viswanathan and I. E. Grossmann. A Combined Penalty Function and OuterApproximation Method for MINLP Optimization. Comput. Chem. Eng., 14(7):769$782,1990$.

[14] J.M. Pinto and I. E. Grossmann. Optimal Cyclic Scheduling of Multistage Continuous Multiproduct Plants. Comput. Chem. Eng., 18(9):797-816, 1994.

[15] B. Finlayson. Nonlinear Analysis in Chemical Engineering. McGraw-Hill, New York, 1980.

[16] J. Villadsen and M.Michelsen. Solution of Differential Equations Models by Polynomial Approximation. Prentice-Hall, 1978.

[17] W. Seider, D. Brengel, A. Provost, and S. Widagdo. Nonlinear Analysis in Process Design. Why Overdesign To Avoid Complex Nonlinearities? Ind. Eng. Chem. Res., 29:805, 1990 .

[18] P. Sistu and B.W. Bequette. Model Predictive Control of Process with Input Multiplicities. Chem. Eng. Sci., 50(6):921-936, 1995.

[19] L.B. Koppel. Input Multiplicities in Nonlinear Multivariable Control Systems. AICHE J., 28(6):935-945, 1982. 
[20] G.A. Hicks and W.H. Ray. Approximation Methods for Optimal Control Synthesis. Can. J. Chem. Eng., 40:522-529, 1971.

[21] V.S. Vassiliadis, R.W.H. Sargent, and C.C. Pantelides. Solution of a class of multistage dynamic optimization problems. 1. problems without path constraints. Ind. Eng. Chem. Res., 33(9):2111-2122, 1994.

[22] A. Flores-Tlacuahuac, L.T. Biegler, and E. Saldívar-Guerra. Dynamic optimization of hips open-loop unstable polymerization reactors. Ind. Eng. Chem. Res., 44(8):2659$2674,2005$.

[23] A. Silva-Beard, A. Flores-Tlacuahuac, and J.C. Arrieta-Camacho. European Symposium on Computer Aided Process Engineering - 12, pages 547-552. Elsevier, 2002.

[24] N. Sahinidis and I.E. Grossmann. MINLP Model for Cyclic Multiproduct Scheduling on Continuous Parallel Lines. Comput. Chem. Eng., 15(2):85-103, 1991.

[25] I.E. Grossmann. Enterprise-wide Optimization: A New Frontier in Process Systems Engineering. AICHE J., 51(7):1846-1857, 2005.

[26] M. Erdirik Dogan and Ignacio E. Grossmann. A Decomposition Method for the Simultaneous Planning and Scheduling of Single Stage Continuous Multiproduct Plants. To appear in Ind.Eng.Chem.Res, 2005. 

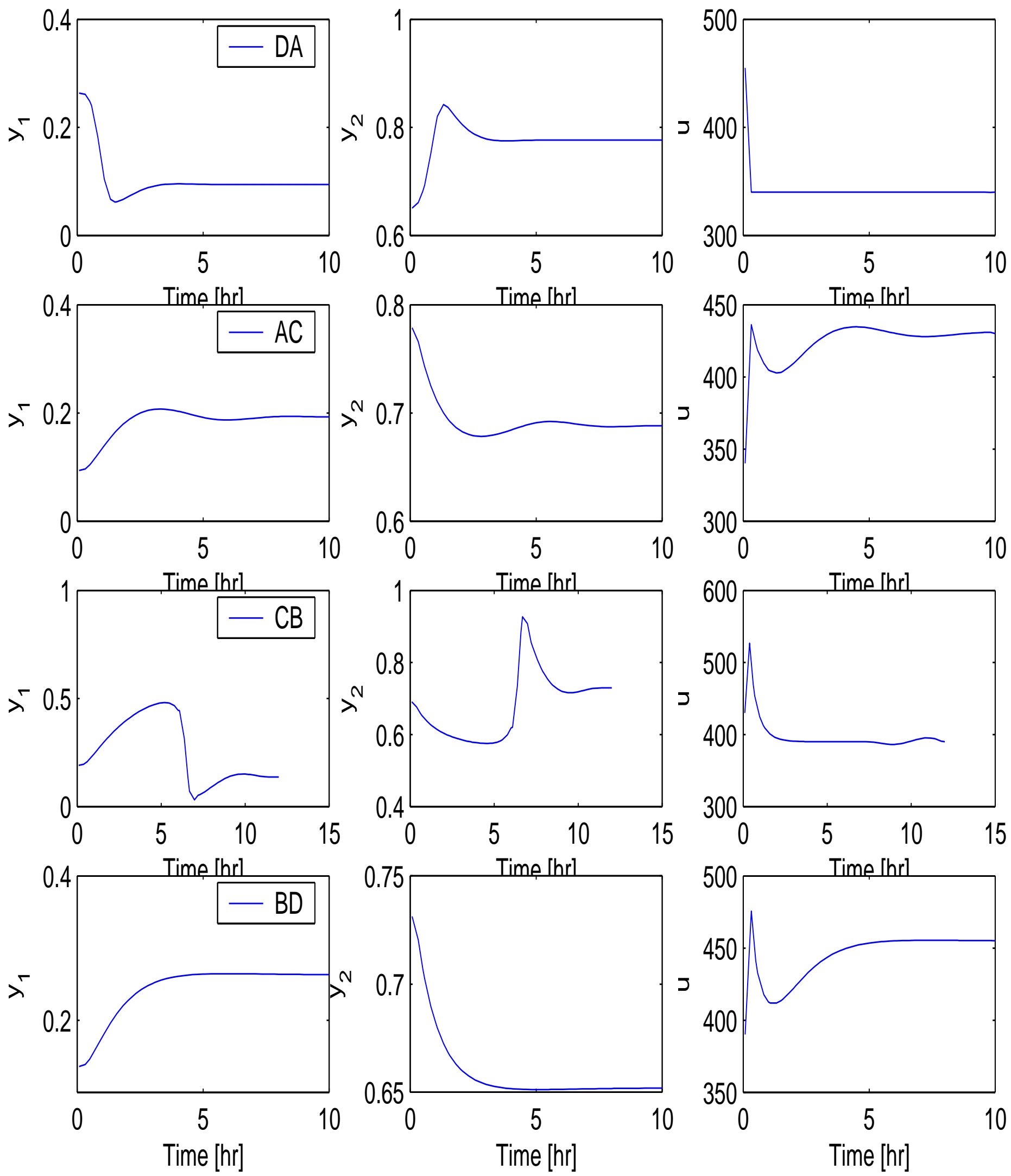

Figure 12: Optimal schedule and dynamic profiles for the third case study, second best solution. 